\title{
EUCLIDEAN JORDAN ALGEBRAS, HIDDEN ACTIONS, AND J-KEPLER PROBLEMS
}

\author{
GUOWU MENG
}

\begin{abstract}
For a simple euclidean Jordan algebra, let co be its conformal algebra, $\mathscr{P}$ be the manifold consisting of its semi-positive rank-one elements, $C^{\infty}(\mathscr{P})$ be the space of complex-valued smooth functions on $\mathscr{P}$. An explicit action of $\mathfrak{c o}$ on $C^{\infty}(\mathscr{P})$, referred to as the hidden action of $\mathfrak{c o}$ on $\mathscr{P}$, is exhibited. This hidden action turns out to be mathematically responsible for the existence of the Kepler problem and its recently-discovered vast generalizations, referred to as J-Kepler problems. The J-Kepler problems are then reconstructed and re-examined in terms of the unified language of euclidean Jordan algebras. As a result, for a simple euclidean Jordan algebra, the minimal representation of its conformal group can be realized either as the Hilbert space of bound states for its J-Kepler problem or as $L^{2}\left(\mathscr{P}, \frac{1}{r} \mathrm{vol}\right)$, where vol is the volume form on $\mathscr{P}$ and $r$ is the inner product of $x \in \mathscr{P}$ with the identity element of the Jordan algebra.
\end{abstract}

\section{INTRODUCTION}

The main message we wish to convey in this paper is that the simple euclidean Jordan algebras introduced by P. Jordan [1] in the 1930's and the various Kepler-type problems we [2] introduced in recent years are intrinsically in one-to-one correspondence: for a simple euclidean Jordan algebra, there is a super integrable model whose configuration space is the manifold consisting of the semi-positive rank-one elements of the Jordan algebra; moreover, the conformal symmetry group of the Jordan algebra is the dynamical symmetry group of the super integrable model. Since they resemble the Kepler/Coulomb problem, these super integrable models are referred to as $\mathbf{J}$-Kepler problems.

The euclidean Jordan algebras were initially introduced by P. Jordan for the purpose of reformulating quantum mechanics in a minimal way. By definition, an euclidean Jordan algebra is a finite dimensional real commutative algebra $V$ with unit such that, for $a, b$ in $V$, 1) $\left.a^{2}(a b)=a\left(a^{2} b\right), 2\right) a^{2}+b^{2}=0$ implies that $a=b=0$. As an example, we have the euclidean Jordan algebra of real symmetric $k \times k$-matrices with the Jordan product being the symmetrized matrix product. A theorem of Jordan, von Neumann and Wigner [3] says that the simple euclidean Jordan algebras consist of four infinity families and one exceptional.

Although physicists quickly lost interest in Jordan algebras, the subsequent further explorations taken on by mathematicians turned out to be quite fruitful. The work of the Koecher school is especially relevant to the Kepler problem. Here is an important discovery made by M. Koecher [4, 5]: simple euclidean Jordan algebras and (irreducible) tube

Date: May 11, 2011.

2000 Mathematics Subject Classification. Primary 22E46, 22E70; Secondary 81S99, 51P05.

Key words and phrases. Euclidean Jordan Algebras, J-Kepler Problems, Hidden Actions, Symmetric Domains.

The author was supported in part by Qiu Shi Science and Technologies Foundation and an internal grant ("Scholol-based Initiatives") from the Hong Kong Univ. of Sci. \& Tech. 
domains are in natural one-to-one correspondence. Combining with our discovery, we conclude that $J$-Kepler problems and (irreducible) tube domains are in natural one-to-one correspondence.

The Kepler problem is a physics problem about two bodies which attract each other by a force inversely proportional to the distance. Mathematically, this is a mechanical system with configuration space $\mathbb{R}_{*}^{3}:=\mathbb{R}^{3} \backslash\{0\}$ and Lagrangian

$$
L=\frac{1}{2} \mathbf{r}^{\prime} \cdot \mathbf{r}^{\prime}+\frac{1}{r}
$$

Here, $\mathbf{r}$ is a function of time $t$ taking value in $\mathbb{R}_{*}^{3}, r=|\mathbf{r}|$ and $\mathbf{r}^{\prime}$ is the time derivative of $\mathbf{r}$. Therefore, quantum mechanically the hamiltonian for the Kepler problem becomes

$$
\hat{H}=-\frac{1}{2} \Delta-\frac{1}{r} \text {. }
$$

Here, $\Delta$ is the Laplace operator on $\mathbb{R}_{*}^{3}$. Physicists are interested in solving the bound state eigenvalue problems for $\hat{H}$, i.e., 1) finding the list of real numbers $\lambda_{0}<\lambda_{1}<\cdots$ such that

$$
\mathscr{H}_{\lambda}:=\left\{\left.\psi \in C^{\infty}\left(\mathbb{R}_{*}^{3}, \mathbb{C}\right)\left|\int_{\mathbb{R}_{*}^{3}}\right| \psi\right|^{2} d^{3} \vec{r}<\infty, \hat{H} \psi=\lambda \psi\right\}
$$

is nontrivial if and only if $\lambda$ is one of $\lambda_{I} ; 2$ ) determining each $\mathscr{H}_{\lambda_{I}}$. It is well known that, for the hamiltonian in Eq. 1.1, the answer is very simple:

$$
\lambda_{I}=-\frac{1 / 2}{(I+1)^{2}}, \quad I=0,1, \ldots
$$

and as a module of $\mathfrak{s o}(4)=\mathfrak{s o}(3) \oplus \mathfrak{s o}(3), \mathscr{H}_{I} \cong V_{I} \otimes V_{I}$, where $V_{I}$ is the $\mathfrak{s o}(3)$-module with dimension $I+1$. What is less well known is a discovery of A.O. Barut and H. Kleinert [6] which essentially says that

$$
\mathcal{H}:=\bigoplus_{I=0}^{\infty} \mathscr{H}_{I}
$$

is a unitary lowest weight $(\mathfrak{s o}(6), \mathrm{SO}(4) \times \mathrm{SO}(2))$-module in the sense of Harish-Chandre [7], hence it can be integrated to a unitary lowest weight module for $\mathrm{SO}_{0}(2,4)$ (the identity component of $\mathrm{SO}(2,4)$ ). Moreover, this module for $\mathrm{SO}_{0}(2,4)$ is minimal in the sense of A. Joesph [8] and also has $L^{2}\left(\mathbb{R}_{*}^{3}, \frac{1}{r} d^{3} \vec{r}\right)$ as a geometric realization.

An apparent mathematical generalization, known to many people, is to replace $\mathbb{R}^{3}$ by $\mathbb{R}^{n}(n \geq 2)$ and keep the hamiltonian in the same form. Similar results are valid: $\lambda_{I}=$ $-\frac{1 / 2}{\left(I+\frac{n-1}{2}\right)^{2}}, \mathscr{H}_{I}$ is an irreducible representation of $\mathrm{SO}(n+1), \bigoplus_{I=0}^{\infty} \mathscr{H}_{I}$ is a unitary lowest weight $(\mathfrak{s o}(n+3), \mathrm{SO}(n+1) \times \mathrm{SO}(2))$-module, hence it can be integrated to a unitary lowest weight module for $\mathrm{SO}_{0}(2, n+1)$ (actually a double of it when $n$ is even); moreover, this module is minimal and also has $L^{2}\left(\mathbb{R}_{*}^{n}, \frac{1}{r} d^{n} \vec{r}\right)$ as a geometric realization.

The less obvious cousins of the Kepler problems were all worked out in recent years. Together with the obvious ones mentioned in the preceding paragraphs, they consist of four infinity families and one exceptional. So it appears that there is a natural one-to-one correspondence between them and the simple euclidean Jordan algebras.

Indeed, this is the case, and here is a quick way to see the one-to-one correspondence. We begin with the notion of Kepler cone for a simple euclidean Jordan algebra. By definition it is the manifold consisting of the rank-one semi-positive elements of the Jordan algebra, equipped with a suitable Riemannian metric. This Kepler cone plays the role of 
$\mathbb{R}_{*}^{3}$. To define the $\mathbf{J}$-Kepler problem, one needs to replace the hamiltonian in Eq. (1.1) by this one:

$$
\hat{h}=-\frac{1}{2} \Delta-\left(\frac{B}{2 r^{2}}+\frac{1}{r}\right) .
$$

Here, $\Delta$ is the (non-positive) Laplace operator on the Kepler cone, $r=r(x)$ is the inner product of $x$ (in the Kepler cone) with the identity element of the Jordan algebra, and $B$ is a constant depending on the Jordan algebra, for example, $B=26$ for the exceptional Jordan algebra. Note that, when the Jordan algebra is the Minkowski space, the J-Kepler problem is equivalent to the Kepler problem, i.e., it is a reformulation of the Kepler problem as a dynamic problem on the open future light cone:

$$
\left\{\left.(t, \mathbf{x})\left|t^{2}=\right| \mathbf{x}\right|^{2}, t>0\right\}
$$

- the Kepler cone in this case.

The J-Kepler problems all share the key features of the Kepler problem, for example, the $I$-th eigenvalue of the hamiltonian is

$$
\lambda_{I}=-\frac{1 / 2}{(I+\rho \delta / 4)^{2}}
$$

here $\rho$ and $\delta$ are the rank and degree of the Jordan algebra respectively. The more detailed results are given in Theorem 5 on page 33

A key mathematical result here is Theorem 1 1 on page 19, which gives an explicit action of the conformal algebra of the Jordan algebra on the space of complex-valued smooth functions on the Kepler cone. In this action, elements of the conformal algebra are realized as differential operators of degree zero, one and two, so this action does not come from an underlying action on the Kepler cone; consequently such an action is referred to as a hidden action of the conformal algebra on the Kepler cone. In our view, this hidden action is the mathematical origin for the J-Kepler problems.

One curious fact, though not presented here, is that the magnetized versions of a given JKepler problem also exist unless the Jordan algebra is the exceptional one. This could lead to some speculations about the fundamental physics. Another fact, which is not proved here either, is that a unitary lowest weight representation can be realized by the Hilbert space of bound states of a magnetized version of a J-Kepler problem if and only if it has the minimal positive Gelfand-Kirillov dimension.

Here is the organization of this paper. In section 2 we give a review of the euclidean Jordan algebras, tailed to our needs. In section 3 , we review the TKK (Tits-Kantor-Koecher) construction [9], something that assigns a simple real Lie algebra (the conformal algebra) to each simple euclidean Jordan algebra. In section 4, we do a bit of structural analysis for the conformal algebra. In section 5, we introduce the notion of Kepler cone for simple euclidean Jordan algebras. In section 6, we introduce the hidden action of the conformal algebra on the Kepler cone, which amounts to the dynamic symmetry of the corresponding J-Kepler problem. In section 7 we introduce the notion of J-Kepler problem for simple euclidean Jordan algebras and show that a J-Kepler problem is just a Kepler-type problem with zero magnetic charge that we introduced and studied in recent years. In section 8, based on the hidden action obtained from section 6, we give the dynamical symmetry analysis and solve the bound state problem for the J-Kepler problem. 
Acknowledgement. We wish to thank the following distinguished mathematicians for providing their moral support for our lonely exploration: M. Atiyah, R. Howe, J.S. Li and C. Taubes.

\section{EUCLIDEAn Jordan Algebras}

The materials reviewed in this and the next sections can be found in Refs. [4, 5]. Recall that an algebra $V$ over a field $\mathbb{F}$ is a vector space over $\mathbb{F}$ together with a $\mathbb{F}$-bilinear map $V \times V \rightarrow V$ which maps $(u, v)$ to $u v$. This $\mathbb{F}$-bilinear map can be recast as a linear map $V \rightarrow \operatorname{End}_{\mathbb{F}}(V)$ which maps $u$ to $L_{u}: v \mapsto u v$.

We say that algebra $V$ is commutative if $u v=v u$ for any $u, v \in V$. As usual, we write $u^{2}$ for $u u$ and $u^{m+1}$ for $u u^{m}$ inductively.

Definition 2.1. A Jordan algebra over $\mathbb{F}$ is just a commutative algebra $V$ over $\mathbb{F}$ such that

$$
\left[L_{u}, L_{u^{2}}\right]=0
$$

for any $u \in V$.

Here are some basic facts about the Jordan algebra $V$ over $\mathbb{F}$ :

- $u^{r} u^{s}=u^{r+s}$ for any $u \in V$ and any integers $r, s \geq 1$.

- $L_{u^{m}}(m \geq 1)$ is a polynomial in $L_{u}$ and $L_{u^{2}}$, for example,

$$
L_{u^{3}}=3 L_{u^{2}} L_{u}-2 L_{u}^{3} .
$$

- For any $u, v, z \in V$, we have

$$
\left[\left[L_{u}, L_{v}\right], L_{z}\right]=L_{u(v z)-v(u z)}
$$

provided that $\operatorname{Char}(\mathbb{F}) \neq 2$.

As the first example, we note that $\mathbb{F}$ is a Jordan algebra over $\mathbb{F}$. Here is a recipe to produce Jordan algebras. Suppose that $\Phi$ is an associative algebra over field $\mathbb{F}$ with characteristic $\neq 2$, and $V \subset \Phi$ is a linear subspace of $\Phi$, closed under square operation, i.e, $u \in V \Rightarrow u^{2} \in V$. Then $V$ is a Jordan algebra over $\mathbb{F}$ under the Jordan product

$$
u v:=\frac{(u+v)^{2}-u^{2}-v^{2}}{2} .
$$

Applying this recipe, we have the following Jordan algebras over $\mathbb{R}$ :

(1) The algebra $\Gamma(n)$. Here $\Phi=\operatorname{Cl}\left(\mathbb{R}^{n}\right)$ 一the Clifford algebra of $\mathbb{R}^{n}$ and $V=$ $\mathbb{R} \oplus \mathbb{R}^{n}$.

(2) The algebra $\mathcal{H}_{n}(\mathbb{R})$. Here $\Phi=M_{n}(\mathbb{R})$-the algebra of real $n \times n$-matrices and $V \subset \Phi$ is the set of symmetric $n \times n$-matrices.

(3) The algebra $\mathcal{H}_{n}(\mathbb{C})$. Here $\Phi=M_{n}(\mathbb{C})$-the algebra of complex $n \times n$-matrices (considered as an algebra over $\mathbb{R}$ ) and $V \subset \Phi$ is the set of Hermitian $n \times n$ matrices.

(4) The algebra $\mathcal{H}_{n}(\mathbb{H})$. Here $\Phi=M_{n}(\mathbb{H})$-the algebra of quaternionic $n \times n$ matrices (considered as an algebra over $\mathbb{R}$ ) and $V \subset \Phi$ is the set of Hermitian $n \times n$-matrices.

The Jordan algebras over $\mathbb{R}$ listed above are special because they can be derived from associated algebras via the above recipe. Let us denote by $\mathcal{H}_{n}(\mathbb{O})$ the algebra for which the underlying real vector space is the set of Hermitian $n \times n$-matrices over octonions $\mathbb{O}$ and the product is the symmetrized matrix product. One can show that $\mathcal{H}_{n}(\mathbb{O})$ is a Jordan algebra if and only if $n \leq 3$. 
Any Jordan algebra $V$ comes with a canonical symmetric bilinear form

$$
\tau(u, v):=\text { the trace of } L_{u v} .
$$

Thanks to Eq. 2.3), $L_{u}$ is self-adjoint with respect to $\tau$.

We say that the Jordan algebra $V$ is semi-simple if the symmetric bilinear form $\tau$ is non-degenerate. We say that the Jordan algebra $V$ is simple if it is semi-simple and has no ideal other than $\{0\}$ and $V$ itself.

By definition, an euclidean Jordan algebra 1 is a real Jordan algebra with an identity element and the positive definite symmetric bilinear form $\tau$. Therefore, an euclidean Jordan algebra is semi-simple and can be uniquely written as the direct sum of simple ideals — ideals which are simple as Jordan algebras.

Theorem 2.1 (Jordan, von Neumann and Wigner). The complete list of simple euclidean Jordan algebras are

(1) The algebra $\Gamma(n)=\mathbb{R} \oplus \mathbb{R}^{n}(n \geq 2)$.

(2) The algebra $\mathcal{H}_{n}(\mathbb{R})(n \geq 3$ or $n=1)$.

(3) The algebra $\mathcal{H}_{n}(\mathbb{C})(n \geq 3)$.

(4) The algebra $\mathcal{H}_{n}(\mathbb{H})(n \geq 3)$.

(5) The algebra $\mathcal{H}_{3}(\mathbb{O})$.

Note that the last one is called the exceptional type because it cannot be obtained from an associative algebra via the recipe we mentioned early. Note also that $\Gamma(1)$ is not simple, $\mathcal{H}_{1}(\mathbb{F})=\mathbb{R}$ is the only associative simple euclidean Jordan algebra, and we have various isomorphisms: $\Gamma(2) \cong \mathcal{H}_{2}(\mathbb{R}), \Gamma(3) \cong \mathcal{H}_{2}(\mathbb{C}), \Gamma(5) \cong \mathcal{H}_{2}(\mathbb{H}), \Gamma(9) \cong \mathcal{H}_{2}(\mathbb{O})$. The first family is called the Dirac type and the next three families are called the hermitian type.

Throughout the remainder of this paper, we always use $V$ to denote a simple euclidean Jordan algebra, and $e$ to denote the identity element of $V$.

The notion of trace is valid for Jordan algebras. For the simple euclidean Jordan algebras, the trace can be easily described: For $\Gamma(n)$, we have

$$
\operatorname{tr}(\lambda, \vec{u})=2 \lambda,
$$

and for all other types, it is the usual trace on matrices.

Recall that $L_{u}: V \rightarrow V$ is the multiplication by $u$ and its trace is denoted by $\operatorname{Tr}\left(L_{u}\right)$. It is a fact that

$$
\frac{1}{\operatorname{dim} V} \operatorname{Tr}\left(L_{u}\right)=\frac{1}{\rho} \operatorname{tr}(u)
$$

where $\rho:=\operatorname{tr}(e)$ is the rank of $V$.

For the inner product on $V$, we take

$$
\langle u \mid v\rangle:=\frac{1}{\rho} \operatorname{tr}(u v)
$$

so that $e$ becomes a unit vector. One can check that $L_{u}$ is self-adjoint with respect to this inner product: $\langle v u \mid w\rangle=\langle v \mid u w\rangle$, i.e., $L_{u}^{\prime}=L_{u}$.

We shall use Dirac's bracket notations: for $u, v \in V$, we declare that $|u\rangle$ is the vector $u,\langle u|$ is the co-vector sending $z \in V$ to $\langle u \mid z\rangle$, and $|u\rangle\langle v|$ is the endomorphism sending $z \in V$ to $\langle v \mid z\rangle u$. Since $L_{u}^{\prime}=L_{u}$, the dual of $L_{u}$, denoted by $L_{u}^{*}$, maps $\langle z|$ to $-\left\langle L_{u} z\right|$.

We shall adopt the following conventions: $x$ is reserved for a point in the smooth space $V$, and $u, v, z, w$ are reserved for vectors in the vector space $V$. We shall fix an orthonomal

\footnotetext{
${ }^{1}$ Called formally real Jordan algebra in the old literature.
} 
basis $\left\{e_{\alpha}\right\}$ for $V$, with respect to which, we can express $x$ as $\sum_{\alpha} x^{\alpha} e_{\alpha}$. For simplicity, we shall write $\sum_{\alpha} e_{\alpha} \frac{\partial}{\partial x^{\alpha}}$ as $\not \partial$.

From here on, we shall also use $V$ to denote the euclidean space with underlying smooth space $V$ and Riemannian metric $d s_{E}^{2}$ :

$$
\begin{aligned}
T_{x} V \times T_{x} V & \rightarrow \mathbb{R} \\
((x, u),(x, v)) & \mapsto\langle u \mid v\rangle .
\end{aligned}
$$

For $u \in V$, we use $\hat{L}_{u}$ to denote the vector field on $V$ whose value at $x \in V$ is $(x,-u x) \in T_{x} V$. Viewed as a differential operator, we have

$$
\hat{L}_{u}=-\langle u x \mid \not \partial\rangle \text {. }
$$

The following theorem is extremely useful when we do concrete computations with Jordan algebras.

Theorem 2.2 (Jordan Frame). Let $V$ be a simple euclidean Jordan algebra of rank $\rho$, $x_{0} \in V$. Suppose that $x_{0}$ is non-zero and satisfies equation $x_{0}^{2}=\operatorname{tr} x_{0} x_{0}$. Then there is an orthogonal basis for $V: e_{11}, \ldots, e_{\rho \rho}, e_{i j}^{\mu}(1 \leq i<j \leq \rho, 1 \leq \mu \leq \delta)$ such that

1) each basis vector has length $\frac{1}{\sqrt{\rho}}$;

2) $e_{i i}^{2}=e_{i i},\left(e_{j k}^{\mu}\right)^{2}=\frac{1}{2}\left(e_{j j}+e_{k k}\right)$;

3) $e_{i i} e_{i j}^{\mu}=e_{j j} e_{i j}^{\mu}=\frac{1}{2} e_{i j}^{\mu}$;

4) $e_{i i} e_{j k}^{\mu}=0$ if $i \notin\{j, k\}$, and $e_{i i} e_{j j}=0$ if $i \neq j$;

5) $e_{11}+\cdots+e_{\rho \rho}=e$;

6) $\operatorname{tr} e_{i i}=1, \operatorname{tr} e_{i j}^{\mu}=0$;

7) $x_{0}$ is a multiple of $e_{11}$.

In the above theorem, $\left\{e_{11}, \ldots, e_{\rho \rho}\right\}$ is called a Jordan frame and the parameter $\delta$ is called the degree of $V$. If $i<j$, we use $V_{i j}$ to denote $\operatorname{span}_{\mathbb{R}}\left\{e_{i j}^{\mu} \mid 1 \leq \mu \leq \delta\right\}-$ the $(i, j)$-Pierce component.

Remark 2.1. Simple euclidean Jordan algebras are isomorphic if and only if they have the same rank $\rho$ and same degree $\delta$, as one can verify from the table below:

\begin{tabular}{|c|c|c|c|c|c|}
\hline$V$ & $\Gamma(n)$ & $\mathcal{H}_{n}(\mathbb{R})$ & $\mathcal{H}_{n}(\mathbb{C})$ & $\mathcal{H}_{n}(\mathbb{H})$ & $\mathcal{H}_{3}(\mathbb{O})$ \\
\hline$\rho$ & 2 & $n$ & $n$ & $n$ & 3 \\
\hline$\delta$ & $n-1$ & 1 & 2 & 4 & 8 \\
\hline
\end{tabular}

It is also clear from this table and the above theorem that, for the simple euclidean Jordan algebras, there is one with rank-one, infinitely many with rank two, four with rank three, and three with rank four or higher.

\section{Tits-KAntor-KoEcher CONSTRUCtion}

The Tits-Kantor-Koecher construction yields a simple real Lie algebras from a simple euclidean Jordan algebra. We begin with the introduction of the Jordan triple product:

$$
\{u v w\}:=u(v w)+w(v u)-(u w) v .
$$

One can check that the Jordan triple product satisfies the following identities:

$$
\begin{aligned}
\{w y z\} & =\{z y w\} \\
\{u v\{z w y\}\} & =\{\{u v z\} w y\}-\{z\{v u w\} y\}+\{z w\{u v y\}\} .
\end{aligned}
$$

For a pair $(u, v) \in V \times V$, we introduce the linear map $S_{u v}: V \rightarrow V$ by declaring that

$$
S_{u v}(z):=\{u v z\} \text {. }
$$


Then Eq. 3.1) is equivalent to the commutation relation

$$
\left[S_{u v}, S_{z w}\right]=S_{\{u v z\} w}-S_{z\{v u w\}} .
$$

Therefore, the span of these $S_{u v}$, denoted by $\mathfrak{s t r}(V)$ or simply $\mathfrak{s t r}$, becomes a real Lie algebra - the structure algebra of $V$. One can check that $S_{u e}=S_{e u}=L_{u}$ and $S_{u v}=$ $\left[L_{u}, L_{v}\right]+L_{u v}$. Then $S_{u v}^{\prime}=S_{v u}$, hence $S_{u v}^{*}(\langle z|)=-\left\langle S_{v u}(z)\right|$. Therefore, in view of Eq. (3.2), we see that $z$ and $w$ in $S_{z w}$ transform under $\mathfrak{s t r}(V)$ as a vector and a co-vector respectively.

Eq. (2.3) amounts to saying that that $\left[L_{u}, L_{v}\right]: V \rightarrow V$ is a derivation; in fact, any derivation is a linear combination of derivations of this form. The derivation algebra of $V$, denoted by $\mathfrak{d e r}(V)$ or simply $\mathfrak{d e r}$, is then a Lie subalgebra of the structure algebra.

The conformal algebra of $V$, denoted by $\mathfrak{c o}(V)$ or simply $\mathfrak{c o}$, is a further extension of $\mathfrak{s t r}(V)$. As a vector space,

$$
\mathfrak{c o}(V)=V \oplus \mathfrak{s t r}(V) \oplus V^{*} .
$$

In the following we shall rewrite $u \in V$ as $X_{u}$ and $\langle v| \in V^{*}$ as $Y_{v}$.

Theorem 3.1 (Koecher). Let $V$ be a simple euclidean Jordan algebra, then $\mathfrak{c o}(V)=$ $V \oplus \mathfrak{s t r}(V) \oplus V^{*}$ becomes a simple real Lie algebra with the defining TKK commutation relations

$$
\begin{gathered}
{\left[X_{u}, X_{v}\right]=0, \quad\left[Y_{u}, Y_{v}\right]=0, \quad\left[X_{u}, Y_{v}\right]=-2 S_{u v},} \\
{\left[S_{u v}, X_{z}\right]=X_{\{u v z\}}, \quad\left[S_{u v}, Y_{z}\right]=-Y_{\{v u z\}},} \\
{\left[S_{u v}, S_{z w}\right]=S_{\{u v z\} w}-S_{z\{v u w\}}}
\end{gathered}
$$

for $u, v, z, w$ in $V$.

It is not hard to remember the TKK commutation relations in the above theorem if one notices that, under the action of the structure algebra, $u$ and $v$ in $S_{u v}$ transform as a vector and a co-vector respectively. It follows from the TKK commutation relations that

$$
\left[D, L_{u}\right]=L_{D u}, \quad\left[D, X_{u}\right]=X_{D u}, \quad\left[D, Y_{u}\right]=Y_{D u}
$$

for any derivation $D \in \mathfrak{d e r}$ and any $u \in V$. In particular, we have $\left[D, L_{e}\right]=0,\left[D, X_{e}\right]=0$ and $\left[D, Y_{e}\right]=0$.

Let $V$ be a simple euclidean Jordan algebra with an orthonormal basis $\left\{e_{\alpha}\right\}$ chosen. Upon recalling the definition of $\not$, we can introduce differential operators

$$
\hat{S}_{u v}=-\left\langle S_{u v}(x) \mid \not \partial\right\rangle, \quad \hat{X}_{u}=-\langle u \mid \not \partial\rangle, \quad \hat{Y}_{v}=-\langle\{x v x\} \mid \not \partial\rangle .
$$

One can verify that $\hat{S}_{u e}=\hat{S}_{e u}=\hat{L}_{u}$ and $\hat{S}_{u v}=\left[\hat{L}_{u}, \hat{L}_{v}\right]+\hat{L}_{u v}$.

The following proposition implies that the conformal algebra of $V$ can be realized as a Lie subalgebra of the Lie algebra of vector fields on $V$.

Proposition 3.1. The TKK commutation relations can be realized by vector fields $\hat{S}_{u v}, \hat{X}_{z}$, $\hat{Y}_{w}$.

Proof. It is clear that $\left[\hat{X}_{u}, \hat{X}_{v}\right]=0$.

$$
\begin{aligned}
{\left[\hat{S}_{u v}, \hat{S}_{z w}\right] } & =\left[-\left\langle S_{u v}(x) \mid \not \partial\right\rangle,-\left\langle S_{z w}(x) \mid \not \partial\right\rangle\right] \\
& =-\left\langle S_{u v} S_{z w}(x) \mid \not \partial\right\rangle+\left\langle S_{z w} S_{u v}(x) \mid \not \partial\right\rangle
\end{aligned}
$$




$$
\begin{aligned}
& =-\left\langle\left[S_{u v}, S_{z w}\right](x) \mid \not \partial\right\rangle \\
& =-\left\langle\left(S_{\{u v z\} w}-S_{z\{v u w\}}\right)(x) \mid \not \partial\right\rangle \\
& =\hat{S}_{\{u v z\} w}-\hat{S}_{z\{v u w\}} \text {. } \\
& {\left[\hat{S}_{u v}, \hat{X}_{z}\right]=\left[-\left\langle S_{u v}(x) \mid \not \partial\right\rangle,-\langle z \mid \not \partial\rangle\right]} \\
& =-\left\langle S_{u v}(z) \mid \not \partial\right\rangle=\hat{X}_{\{u v z\}} \text {. } \\
& {\left[\hat{X}_{u}, \hat{Y}_{v}\right]=[-\langle u \mid \not \partial\rangle,-\langle\{x v x\} \mid \not \partial\rangle]} \\
& =2\langle\{u v x\} \mid \not\rangle=-2 \hat{S}_{u v} \text {. } \\
& {\left[\hat{S}_{u v}, \hat{Y}_{z}\right]=\left[-\left\langle S_{u v}(x) \mid \not \partial\right\rangle,-\langle\{x z x\} \mid \not \partial\rangle\right]} \\
& =2\left\langle\left\{S_{u v}(x) z x\right\} \mid \not \partial\right\rangle-\left\langle S_{u v}(\{x z x\}) \mid \not \partial\right\rangle \\
& =2\left\langle S_{x z} S_{u v}(x) \mid \not \partial\right\rangle-\left\langle S_{u v} S_{x z}(x) \mid \not \partial\right\rangle \\
& =\left\langle S_{x z} S_{u v}(x) \mid \not \partial\right\rangle-\left\langle\left[S_{u v}, S_{x z}\right](x) \mid \not \partial\right\rangle \\
& =\left\langle S_{x z} S_{u v}(x)-S_{\{u v x\} z}(x) \mid \not \partial\right\rangle+\left\langle S_{x\{v u z\}}(x) \mid \not \partial\right\rangle \\
& =-\hat{Y}_{\{v u z\}} \text {. }
\end{aligned}
$$

Finally,

$$
\begin{aligned}
{\left[\hat{Y}_{u}, \hat{Y}_{v}\right] } & =[-\langle\{x u x\} \mid \not \partial\rangle-\langle\{x v x\} \mid \not \partial\rangle] \\
& =2\langle\{\{x u x\} v x\} \mid \not\rangle-<u \leftrightarrow v\rangle \\
& =2\left\langle\left[S_{x v}, S_{x u}\right] x \mid \not \partial\right\rangle \\
& =\left\langle\left[S_{x v}, S_{x u}\right] x \mid \not \partial\right\rangle-<u \leftrightarrow v> \\
& =\left\langle\left(S_{\{x v x\} u}-S_{x\{v x u\}}\right) x \mid \not \partial\right\rangle-<u \leftrightarrow v> \\
& =\left\langle S_{\{x v x\}} x\right| \not \partial-\langle u \leftrightarrow v>\text { because }\{u x v\}=\{v x u\} \\
& =-\left\langle\left[S_{x v}, S_{x u}\right] x \mid \not \partial\right\rangle \\
& =0,
\end{aligned}
$$

because it is equal to the negative half of itself. Here, $<u \leftrightarrow v>$ means the term same as the one on the left except that $u$ and $v$ are interchanged.

We conclude this section with a few more terminologies and notations. Let $\Omega$ be the symmetric cone of $V$ and $\operatorname{Str}(V)$ be the structure group of $V$. By definition, $\Omega$ is the topological interior of $\left\{x^{2} \mid x \in V\right\}$ and

$$
\operatorname{Str}(V)=\left\{g \in G L(V) \mid P(g x)=g P(x) g^{\prime} \quad \forall x \in V\right\} .
$$

Here $P(x):=2 L_{x}^{2}-L_{x^{2}}$ and it is called the quadratic representation of $x$.

We write $V^{\mathbb{C}}$ for the complexification of $V$, denote by $T_{\Omega}$ the tube domain associated with $V$. By definition, $T_{\Omega}=V \oplus i \Omega-$ a domain inside $V^{\mathbb{C}}$. We say that map $f$ : $T_{\Omega} \rightarrow T_{\Omega}$ is a holomorphic automorphism of $T_{\Omega}$ if $f$ is invertible and both $f$ and $f^{-1}$ are holomorphic. We use Aut $\left(T_{\Omega}\right)$ to denote the group of holomorphic automorphisms of $T_{\Omega}$.

It is a fact that both $\operatorname{Str}(V)$ and $\operatorname{Aut}\left(T_{\Omega}\right)$ are Lie groups. The Lie algebra of $\operatorname{Str}(V)$ is $\mathfrak{s t r}(V)$, the Lie algebra of $\operatorname{Aut}\left(T_{\Omega}\right)$ is $\mathfrak{c o}(V)$, and its universal enveloping algebra is called the TKK algebra of $V$. The simply connected Lie group with $\mathfrak{c o}$ as its Lie algebra, denoted by $\mathrm{Co}(V)$ or simply $\mathrm{Co}$, shall be referred to as the conformal group of $V$. While the structure algebra is reductive, the conformal algebra is simple. 


\section{Cantan Involutions and Vogan Diagrams}

The complex simple Lie algebras are completely classified by (connected) Dynkin diagrams. The real simple Lie algebras are completely classified too, and can be represented by Vogan diagrams - Dynkin diagrams with some extra information on the Dynkin nodes.

4.1. Generalities. Here is a quick review of real simple Lie algebras and Vogan diagrams. Let $\mathfrak{g}$ be a real simple Lie algebra, and $\langle$,$\rangle be its Killing form. An involution \theta$ on $\mathfrak{g}$ is called a Cartan involution if the bilinear form $(X, Y) \mapsto\langle X, \theta(Y)\rangle$ is negative definite. Given a Cartan involution $\theta$, we have the corresponding Cartan decomposition:

$$
\mathfrak{g}=\mathfrak{u} \oplus \mathfrak{p} .
$$

Here, $\mathfrak{u}$ ( $\mathfrak{p}$ resp.) is the eigenspace of $\theta$ with eigenvalue 1 ( -1 resp.). A subalgebra $\mathfrak{h}$ of $\mathfrak{g}$ is called a $\theta$-stable Cartan subalgebra of $\mathfrak{g}$ if $\mathfrak{h}^{\mathbb{C}}$ is a Cartan algebra of $\mathfrak{g}^{\mathbb{C}}$ and $\theta(\mathfrak{h})=\mathfrak{h}$. Here, $\mathfrak{h}^{\mathbb{C}}$ ( $\mathfrak{g}^{\mathbb{C}}$ resp.) is the complexification of $\mathfrak{h}(\mathfrak{g}$ resp.).

Here are some basic facts:

- There is a Cartan involution $\theta$ on $\mathfrak{g}$, unique up to conjugations.

- $\operatorname{span}_{\mathbb{R}}\{X+i Y \mid X \in \mathfrak{u}, Y \in \mathfrak{p}\}$ is a compact Lie algebra.

- $\theta$-stable Cartan subalgebra of $\mathfrak{g}$ exists, but are not all conjugate to each other.

Given a $\theta$-stable Cartan subalgebra $\mathfrak{h}$, there is a corresponding root space decomposition:

$$
\mathfrak{g}^{\mathbb{C}}=\mathfrak{h}^{\mathbb{C}} \oplus \bigoplus_{\alpha \in \Delta} \mathfrak{g}_{\alpha}
$$

A root $\alpha$ w.r.t. $\left(\mathfrak{g}^{\mathbb{C}}, \mathfrak{h}^{\mathbb{C}}\right)$ is called compact (non-compact resp.) if $\mathfrak{g}_{\alpha}$ is a subspace of $\mathfrak{u}^{\mathbb{C}}$ $\left(\mathfrak{p}^{\mathbb{C}}\right.$ resp.). Here is a simple fact on compact or non-compact roots: suppose that root $\alpha$ is either compact or non-compact, then one can choose root vectors $E_{\alpha} \in \mathfrak{g}_{\alpha}, E_{-\alpha} \in \mathfrak{g}_{-\alpha}$ with both $\sqrt{-1}\left(E_{\alpha}+E_{-\alpha}\right)$ and $E_{\alpha}-E_{-\alpha}$ in $\mathfrak{g}$, and an element $H_{\alpha}$ in $\sqrt{-1} \mathfrak{h}$, such that

$$
\left[H_{\alpha}, E_{ \pm \alpha}\right]= \pm 2 E_{ \pm \alpha}, \quad\left[E_{\alpha}, E_{-\alpha}\right]=\left\{\begin{array}{cc}
H_{\alpha} & \text { if } \alpha \text { is compact } \\
-H_{\alpha} & \text { if } \alpha \text { is non-compact. }
\end{array}\right.
$$

We say that a $\theta$-stable Cartan subalgebra of $\mathfrak{g}$ is maximally compact if $\operatorname{dim}(\mathfrak{h} \cap \mathfrak{u})$ is as large as possible. To get a Vogan diagram, the first step is to find a maximally compact $\theta$ stable Cartan subalgebra $\mathfrak{h}$ for $\mathfrak{g}$. The next step is to chose a simple root system $R$ (or Weyl chamber) for the corresponding root system $\Delta$. Such a $R$ is unique up to the action by the Weyl group $W(\Delta)$. Since $\mathfrak{h}$ has been chosen to be maximally compact, the roots w.r.t. $\left(\mathfrak{g}^{\mathbb{C}}, \mathfrak{h}^{\mathbb{C}}\right)$ never vanish on $\mathfrak{h} \cap \mathfrak{u}$, hence are either complex-valued or imaginary-valued on $\mathfrak{h}$. So $R$ splits into two classes: complex and imaginary. Since $R$ is invariant under complex conjugation, the class of complex simple roots splits further into various conjugate pairs of simple roots. Since the corresponding root space $\mathfrak{g}_{\alpha}$ for an imaginary root $\alpha$ is a subspace of $\mathfrak{u}^{\mathbb{C}}$ or $\mathfrak{p}^{\mathbb{C}}$, the class of imaginary simple roots splits further into two subclasses: compact and non-compact.

Definition 4.1. By definition, a Vogan diagram is a Dynkin diagram with such an information about its nodes recorded: we paint each imaginary noncompact node black, connect each conjugate pair of complex nodes by a two-way arrow, and do nothing to the imaginary compact nodes.

\footnotetext{
${ }^{2}$ In this convention, $H_{\alpha} \in \sqrt{-1} \mathfrak{h}$ is fixed by condition $\alpha\left(H_{\alpha}\right)=2, E_{\alpha}$ is unique up to a sign and $E_{-\alpha}$ is fixed once $E_{\alpha}$ is fixed.
} 
Note that one can recover the simple real Lie algebra from one of its Vogan diagrams. Note also that, in the equal rank case (i.e., the case when $\mathfrak{g}$ and $\mathfrak{u}$ have the same rank), $\mathfrak{h} \subset \mathfrak{u}$, so every root is either compact or non-compact, and there is no conjugate pair of Dynkin nodes.

4.2. Analysis for the conformal algebra. The conformal algebra is a real simple Lie algebra per theorem 3.1 so it admits a Cartan involution $\theta$, unique up to inner automorphisms. Indeed, one can choose $\theta$ such that

$$
\theta\left(X_{u}\right)=Y_{u}, \quad \theta\left(Y_{u}\right)=X_{u}, \quad \theta\left(S_{u v}\right)=-S_{v u} .
$$

The resulting Cartan decomposition is $\mathfrak{c o}=\mathfrak{u} \oplus \mathfrak{p}$ with

$$
\mathfrak{u}=\operatorname{span}_{\mathbb{R}}\left\{\left[L_{u}, L_{v}\right], X_{w}+Y_{w} \mid u, v, w \in V\right\}, \quad \mathfrak{p}=\operatorname{span}_{\mathbb{R}}\left\{L_{u}, X_{v}-Y_{v} \mid u, v \in V\right\} .
$$

Note that $\mathfrak{u}$ is reductive with center spanned by $X_{e}+Y_{e}$ and its semi-simple part $\overline{\mathfrak{u}}$ is

$$
\operatorname{span}_{\mathbb{R}}\left\{\left[L_{u}, L_{v}\right], X_{w}+Y_{w} \mid u, v, w \in(\mathbb{R} e)^{\perp}\right\} .
$$

Sometime we need to emphasize the dependence on $V$, then we rewrite $\mathfrak{u}$ as $\mathfrak{u}(V)$. It is a fact that $\mathfrak{s t r}$ and $\mathfrak{u}$ are different real forms of the same complex reductive Lie algebra. In fact, one can identify their complexfications as follows:

$$
\left[L_{u}, L_{v}\right] \leftrightarrow\left[L_{u}, L_{v}\right], \quad-\frac{i}{2}\left(X_{w}+Y_{w}\right) \leftrightarrow L_{w}
$$

Now we have another natural chain of real Lie algebras associated with the Jordan algebra $V$ :

$$
\mathfrak{d e r} \subset \overline{\mathfrak{u}} \subset \mathfrak{u} \subset \mathfrak{c o} .
$$

We shall use $\tilde{U}$ to denote the closed Lie subgroup of Co whose Lie algebra is $\mathfrak{u}$. Note that $\tilde{U}$ is a closed maximal subgroup of Co with $\tilde{U} / \mathrm{Z}$ is compact. (Here $\mathrm{Z}$ is the center of Co.)

Here is a detailed summary of all real Lie algebras we have encountered:

\begin{tabular}{|c|c|c|c|c|}
\hline$V$ & $\mathfrak{d e r}$ & $\mathfrak{s t r}$ & $\mathfrak{u}$ & $\mathfrak{c o}$ \\
\hline$\Gamma(n)$ & $\mathfrak{s o}(n)$ & $\mathfrak{s o}(n, 1) \oplus \mathbb{R}$ & $\mathfrak{s o}(n+1) \oplus \mathfrak{s o}(2)$ & $\mathfrak{s o}(n+1,2)$ \\
\hline $\mathcal{H}_{n}(\mathbb{R})$ & $\mathfrak{s o}(n)$ & $\mathfrak{s l}(n, \mathbb{R}) \oplus \mathbb{R}$ & $\mathfrak{u}(n)$ & $\mathfrak{s p}(n, \mathbb{R})$ \\
\hline $\mathcal{H}_{n}(\mathbb{C})$ & $\mathfrak{s u}(n)$ & $\mathfrak{s l}(n, \mathbb{C}) \oplus \mathbb{R}$ & $\mathfrak{s u}(n) \oplus \mathfrak{s u}(n) \oplus \mathfrak{u}(1)$ & $\mathfrak{s u}(n, n)$ \\
\hline $\mathcal{H}_{n}(\mathbb{H})$ & $\mathfrak{s p}(n)$ & $\mathfrak{s u}(2 n) \oplus \mathbb{R}$ & $\mathfrak{u}(2 n)$ & $\mathfrak{s o}^{*}(4 n)$ \\
\hline $\mathcal{H}_{3}(\mathbb{O})$ & $\mathfrak{f}_{4}$ & $\mathfrak{e}_{6(-26)} \oplus \mathbb{R}$ & $\mathfrak{e}_{6} \oplus \mathfrak{s o}(2)$ & $\mathfrak{e}_{7}(-25)$ \\
\hline
\end{tabular}

To get a Vogan diagram for $\mathfrak{c}$, the first step is to find a maximally compact $\theta$-stable Cartan subalgebra $\mathfrak{h}$ for $\mathfrak{c o}$. Since we are in the equal rank case, $\mathfrak{h} \subset \mathfrak{u}$, and a root $\alpha$ is either compact or non-compact. Recall that $e_{11}$ denotes the first element of a Jordan frame for $V$.

Lemma 4.1. There is a maximally compact $\theta$-stable Cartan subalgebra $\mathfrak{h}$ for $\mathfrak{c o}$, with respect to which, there is a simple root system consisting of imaginary roots $\alpha_{0}, \alpha_{1}, \ldots$, $\alpha_{r}$ such that, for $i \geq 1, \alpha_{i}$ is compact with $H_{\alpha_{i}}, E_{ \pm \alpha_{i}} \in \overline{\mathfrak{u}}^{\mathbb{C}}$, and $\alpha_{0}$ is non-compact with

$$
H_{\alpha_{0}}=i\left(X_{e_{11}}+Y_{e_{11}}\right), \quad E_{ \pm \alpha_{0}}=\frac{i}{2}\left(X_{e_{11}}-Y_{e_{11}}\right) \mp L_{e_{11}} .
$$

Proof. Let us fix a Jordan frame $\left\{e_{i i} \mid 1 \leq i \leq \rho\right\}$. Since $\mathfrak{u}$ is compact, being an abelian subalgebra of $\mathfrak{u}, \mathfrak{h}^{\prime}:=\operatorname{span}_{\mathbb{R}}\left\{X_{e_{i i}}+Y_{e_{i i}} \mid 1 \leq i \leq \rho\right\}$ can be extended to a Cartan subalgebra $\mathfrak{h}$ for $\mathfrak{u}$, hence a maximally compact $\theta$-stable Cartan subalgebra $\mathfrak{h}$ for $\mathfrak{c}$.

Under the action of $\mathfrak{h}$, we have decomposition $\mathfrak{c o}^{\mathbb{C}}=\mathfrak{u}^{\mathbb{C}} \oplus \mathfrak{p}^{\mathbb{C}}$. Therefore, if $\alpha$ is a root for $\left(\mathfrak{c o}^{\mathbb{C}}, \mathfrak{h}^{\mathbb{C}}\right), \mathfrak{g}_{\alpha}$ is a subset of either $\mathfrak{u}^{\mathbb{C}}$ or $\mathfrak{p}^{\mathbb{C}}$, so $\alpha$ is either compact or non-compact. 
To understand the non-compact roots for $\left(\mathfrak{c o}^{\mathbb{C}}, \mathfrak{h}^{\mathbb{C}}\right)$, we introduce

$$
E_{u}^{ \pm}=\frac{\sqrt{-1}}{2}\left(X_{u}-Y_{u}\right) \mp L_{u}, \quad h_{u}=\sqrt{-1}\left(X_{u}+Y_{u}\right), \quad u \in V .
$$

and verify that

$$
\left\{\begin{array}{l}
{\left[h_{u}, E_{v}^{ \pm}\right]= \pm 2 E_{u v}^{ \pm}, \quad\left[E_{u}^{+}, E_{v}^{-}\right]=-h_{u v}-2\left[L_{u}, L_{v}\right]} \\
{\left[E_{u}^{+}, E_{v}^{+}\right]=\left[E_{u}^{-}, E_{v}^{-}\right]=0, \quad\left[h_{u}, h_{v}\right]=4\left[L_{u}, L_{v}\right] .}
\end{array}\right.
$$

The first identity in Eq. (4.3) implies that, under the action of $\mathfrak{h}^{\prime}$, we have the following decomposition of $\mathfrak{p}^{\mathbb{C}}$ :

$$
\mathfrak{p}^{\mathbb{C}}=\bigoplus_{i \leq j}\left(\mathfrak{g}_{i j}^{+} \oplus \mathfrak{g}_{i j}^{-}\right)
$$

where $\mathfrak{g}_{i i}^{ \pm}=\operatorname{span}_{\mathbb{C}}\left\{E_{e_{i i}}^{ \pm}\right\}$and $\mathfrak{g}_{i j}^{ \pm}=\operatorname{span}_{\mathbb{C}}\left\{E_{u}^{ \pm} \mid u \in V_{i j}\right\}$ if $i<j$.

Let $\alpha$ be a non-compact root. The decomposition above implies that $\mathfrak{g}_{\beta} \subset \mathfrak{g}_{i j}^{ \pm}$for some $i, j$ with $i \leq j$. Since $\mathfrak{g}_{i i}^{ \pm}$is one-dimensional and Eq. (4.3) implies that

$$
\left[E_{e_{i i}}^{+}, E_{e_{i i}}^{-}\right]=-h_{e_{i i}}, \quad\left[h_{e_{i i}}, E_{e_{i i}}^{ \pm}\right]= \pm 2 E_{e_{i i}}^{ \pm},
$$

we conclude that, in view of Eq. 4.1, there is a non-compact root $\beta_{i}$ such that $\mathfrak{g}_{ \pm \beta_{i}}=\mathfrak{g}_{i i}^{ \pm}$ and $H_{\beta_{i}}=h_{e_{i i}}$. We call $\beta_{i}$ a non-compact root of type $I$.

Suppose that $\alpha$ is a non-compact root of type II, i.e., not of type I. We may assume that $\mathfrak{g}_{\alpha} \subset \mathfrak{g}_{j k}^{+}$for some $j<k$, then the root vector $E_{\alpha} \in \mathfrak{g}_{\alpha}$ is of the form

$$
E_{\alpha}=E_{z}^{+}
$$

for some $z \in V_{j k}^{\mathbb{C}}$. Consequently, $E_{-\alpha}=E_{\bar{z}}^{-}$, and Eq. (4.3) implies that

$$
\left[E_{\alpha}, E_{-\alpha}\right]=-h_{z \bar{z}}-2\left[L_{z}, L_{\bar{z}}\right] \text {. }
$$

So there is a non-compact root $\beta_{j k}^{z}$ such that $\mathfrak{g}_{ \pm \beta_{j k}^{z}} \subset \mathfrak{g}_{j k}^{ \pm}$and $H_{\beta_{j k}^{z}}=h_{z \bar{z}}+2\left[L_{z}, L_{\bar{z}}\right]$.

Of course, there are exactly $\delta$-many such $z$.

In summary, the non-compact roots are

$$
\pm \beta_{i} \text { and } \pm \beta_{j k}^{z}
$$

and

$$
\mathfrak{g}_{\beta_{i}}, \mathfrak{g}_{\beta_{j k}^{z}} \subset \mathfrak{p}_{+}^{\mathbb{C}}:=\bigoplus_{i \leq j} \mathfrak{g}_{i j}^{+} .
$$

To continue the proof, with the help of Eq. 4.3, we observe that

$$
\beta_{1 i}^{z}\left(H_{\beta_{i}}\right)=\beta_{1 i}^{z}\left(H_{\beta_{1}}\right)=1>0, \quad \beta_{1 i}^{z}\left(H_{\beta_{1 i}^{z}}\right)=2>0, \quad \beta_{1 j}^{z}\left(H_{\beta_{j k}^{w}}\right)>0 .
$$

Here, only the last inequality is not clear, but it can be verified by a case-by-case study ${ }^{3}$. This observation implies that, for $\alpha=\beta_{i}$ or $\beta_{j k}^{z}, H_{\beta_{1}}$ and $H_{-\alpha}$ cannot be in the same Weyl chamber, i.e., $\beta_{1}$ and $-\alpha$ cannot be in the same simple root system.

Fixing a simple root system containing the non-compact root $\beta_{1}$, all we need to do is to show that this simple root system cannot contain another non-compact root. Otherwise, this simple system consisted of simple roots $\gamma_{1}, \ldots, \gamma_{t}, \delta_{1}, \ldots, \delta_{s}$ with $\delta_{i}$ compact, $\gamma_{j}$ non-compact, $t>1, \gamma_{1}=\beta_{1}$ and each $\gamma_{j}$ is either of the form $\beta_{i}$ or of the form $\beta_{k l}^{z}$ due to the conclusion in the previous paragraph, so the first identity in Eq. 4.3) implies that,

$$
\gamma_{j}\left(h_{e}\right)=2, \quad 1 \leq j \leq t
$$

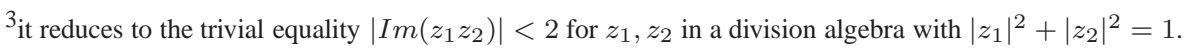


Since the rank of $\mathfrak{c o}$ is one more than the rank of $\overline{\mathfrak{u}}, s$ is less than the rank of $\overline{\mathfrak{u}}$, so there is a compact root $\lambda$ such that

$$
\lambda=\sum_{i} m_{i} \gamma_{i}+\sum_{j} n_{j} \delta_{j}
$$

for some non-negative integers $n_{j}$ and $m_{i}$ with $\sum_{i} m_{i} \neq 0$. Since $\alpha\left(h_{e}\right)$ is equal to zero if $\alpha$ is compact, we have a contradiction:

$$
0=\lambda\left(h_{e}\right)=2 \sum_{i} m_{i} .
$$

As a corollary of the above lemma, the Vogan diagram we have arrived at for the conformal algebra of a simple euclidean Jordan algebra has no complex nodes and only one node painted black. Here is a pictorial summary of the Vogan diagrams for the conformal

\begin{tabular}{|c|c|}
\hline $\mathfrak{c o}$ & Vogan Diagram \\
\hline $\mathfrak{s o}(2,2 n)$ & -- \\
\hline $\mathfrak{s o}(2,2 n+1)$ & $\circ \circ--\propto \Longrightarrow 0$ \\
\hline $\mathfrak{s p}(n, \mathbb{R})$ & $\circ-0--\infty \perp$ \\
\hline $\mathfrak{s u}(n, n)$ & $0--0 \longrightarrow 0--0$ \\
\hline $\mathfrak{s o}^{*}(4 n)$ & $0--c$ \\
\hline $\mathfrak{e}_{7(-25)}$ & \\
\hline
\end{tabular}
algebras:

\section{Kepler Cones}

The goal in this section is to introduce the Kepler cone, an open Riemannian manifold which serves as the configuration space for the J-Kepler problem.

Definition 1 (Kepler Cone). Let $V$ be a simple euclidean Jordan algebra. The Kepler cone is a Riemannian manifold whose underlying smooth manifold is

$$
\mathscr{P}:=\left\{x \in V \mid x^{2}=(\operatorname{tr} x) x, \operatorname{tr} x>0\right\}
$$


and its Riemannian metric, referred to as the Kepler metric, is the restriction of

$$
d s_{K}^{2}:=\frac{2}{\rho} d s_{E}^{2}-(d\langle e \mid x\rangle)^{2}
$$

from $V$ to $\mathscr{P}$.

We shall also use $\mathscr{P}$ to denote the Kepler cone. By introducing coordinates, it is not hard to see that the Kepler cone is a smooth real affine variety.

$\mathscr{P}$ is called the Kepler cone because it is isometric to the open geometric cone over projective space

$$
\mathbb{P}:=\{x \in \mathscr{P} \mid \operatorname{tr}(x)=\sqrt{2 \rho}\} .
$$

Here, as Riemannian manifolds, $\mathscr{P}$ is viewed as $\left(\mathscr{P}, d s_{K}^{2} \mid \mathscr{P}\right), \mathbb{R}_{+} \times \mathbb{P}$ is viewed as $\left(\mathbb{R}_{+} \times \mathbb{P}, d r^{2}+r^{2} d s_{E}^{2} \mid \mathbb{P}\right)$, and the isometry is

$$
\begin{aligned}
\iota: \mathscr{P} & \longrightarrow \mathbb{R}_{+} \times \mathbb{P} \\
x & \mapsto\left(\frac{\operatorname{tr} x}{\rho}, \sqrt{2} \frac{x}{|x|}\right) .
\end{aligned}
$$

Note that, being the intersection of $\mathscr{P}$ with the sphere of radius $\sqrt{2}$ and centered at the origin of $V, \mathbb{P}$ is a compact symmetric space of rank-one:

\begin{tabular}{|l|l|l|l|l|c|}
\hline$V$ & $\Gamma(n)$ & $\mathcal{H}_{n}(\mathbb{R})$ & $\mathcal{H}_{n}(\mathbb{C})$ & $\mathcal{H}_{n}(\mathbb{H})$ & $\mathcal{H}_{3}(\mathbb{O})$ \\
\hline $\mathbb{P}$ & $\mathrm{S}^{n-1}$ & $\mathbb{R} P^{n-1}$ & $\mathbb{C} P^{n-1}$ & $\mathbb{H} P^{n-1}$ & $\mathbb{O} P^{2}$ \\
\hline
\end{tabular}

One can check that the Riemannian metric $d s_{\mathbb{P}}^{2}$ on projective space $\mathbb{P}$ is the round metric of the unit spheres for the Dirac type and is four times the Fubini-Study metric

$$
d s_{F S}^{2}=\frac{|d Z|^{2}}{|Z|^{2}}-\frac{|Z \cdot d \bar{Z}|^{2}}{|Z|^{4}}
$$

of projective spaces for the other types.

We conclude this section with a technical lemma.

Lemma 5.1. Let $V$ be a simple euclidean Jordan algebra with rank $\rho$ and degree $\delta$, and $r=\langle e \mid x\rangle$.

i) Let $e_{\alpha}$ be an orthonormal basis for $V$, then

$$
\frac{\sum_{\alpha, \beta}\left|\left[L_{e_{\alpha}}, L_{e_{\beta}}\right] x\right\rangle\left\langle\left[L_{e_{\alpha}}, L_{e_{\beta}}\right] x\right|}{\frac{\rho^{2}}{2}\left(1+\frac{\delta}{4}(\rho-2)\right)}=r \sum_{\alpha}\left|e_{\alpha}\right\rangle\left\langle e_{\alpha} x|-| x\right\rangle\langle x|
$$

for any $x \in \mathscr{P}$.

ii) For each $u \in V$ and each $x \in \mathscr{P}$, the value of $\hat{L}_{u}$ at $x$ is a tangent vector of $\mathscr{P}$ at $x$. So $\hat{L}_{u}$ descends to a differential operator on the Kepler cone.

iii) Let $\lambda_{u}=\frac{(\rho / 2-1) \delta}{2} \frac{\langle u \mid x\rangle}{r}+\frac{\rho \delta}{4}\langle u \mid e\rangle$, vol $\mathscr{P}$ be the volume element on $\mathscr{P}$, and $\mathscr{L}_{u}$ be the Lie derivative with respect to vector field $\hat{L}_{u}$ on the Kepler cone. Then

$$
\mathscr{L}_{u}\left(\frac{1}{r} \operatorname{vol}_{\mathscr{P}}\right)=-2 \lambda_{u} \frac{1}{r} \operatorname{vol} \mathscr{P} .
$$

Consequently, $\tilde{L}_{u}:=\hat{L}_{u}-\lambda_{u}$ is a skew-hermitian operator with respect to inner product

$$
\left(\psi_{1}, \psi_{2}\right):=\int_{\mathscr{P}} \overline{\psi_{1}} \psi_{2} \frac{1}{r} \operatorname{vol} \mathscr{P}
$$

for compactly-supported smooth functions on $\mathscr{P}$. 
Proof. i) Since both sides of the identity are homogeneously quadratic in $x$, one may assume that $\operatorname{tr} x=1$. Choosing a Jordan frame $\left\{e_{11}, \ldots, e_{\rho \rho}\right\}$ with $e_{11}=x$ and an associated orthonormal basis for $V$, the detailed proof then becomes just a straightforward computation, so we skip it.

ii) Let $x_{0} \in \mathscr{P}$. Since $x_{0}^{2}=\operatorname{tr} x_{0} x_{0}$ and $\operatorname{tr} x_{0}>0$, one can write $x_{0}=\operatorname{tr} x_{0} e_{11}$ so that $e_{11}^{2}=e_{11}$ and $\operatorname{tr} e_{11}=1$. Extending $e_{11}$ to a Jordan frame $\left\{e_{11}, \ldots, e_{\rho \rho}\right\}$ for $V$, then we can decompose $V$ orthogonally into the direct sum of the Pierce components $V_{i j}(i \leq j)$. Then

$$
u x_{0} \in \bigoplus_{j=1}^{\rho} V_{1 j} .
$$

By linearizing equation $x^{2}=\operatorname{tr} x x$ at $x_{0}$, it is clear that the tangent space of $\mathscr{P}$ at $x_{0}$, when translated to 0 , is exactly $\bigoplus_{j=1}^{\rho} V_{1 j}$. Therefore,

$$
\left.\hat{L}_{u}\right|_{x_{0}}=\left(x_{0},-u x_{0}\right) \in T_{x_{0}} \mathscr{P} .
$$

(In fact, one can show that the structure group of $V$ acts on $\mathscr{P}$ transitively.)

iii) We wish to prove identity (5.6) at $x_{0} \in \mathscr{P}$. To do that we need to choose a local coordinate system for $\mathscr{P}$ around $x_{0}$ and do the computations. We may choose a Jordan frame $\left\{e_{11}, \ldots, e_{\rho \rho}\right\}$ such that $x_{0}=a e_{11}$ for some $a>0$. Write

$$
x=\sum_{i=1}^{\rho} x_{i i} e_{i i}+\sum_{1 \leq i<j \leq \rho}^{1 \leq \mu \leq \delta} x_{i j}^{\mu} e_{i j}^{\mu},
$$

by solving equation $x^{2}=\operatorname{tr} x x$, we know that $x_{11}, x_{1 j}^{\alpha}$ 's are independent real variables and the Taylor expansion of the other variables starts at quadratic terms in $\left(x_{11}-a\right), x_{1 j}^{\alpha}$ 's. Therefore,

$$
d s_{K}^{2}=\frac{1}{\rho^{3}}\left[\left(d x_{11}\right)^{2}+2 \sum_{2 \leq j \leq \rho}^{1 \leq \alpha \leq \delta}\left(d x_{1 j}^{\alpha}\right)^{2}\right]+O\left(\left|x-x_{0}\right|^{2}\right)
$$

and

$$
\operatorname{vol}_{\mathscr{P}}=b d x_{11} \wedge\left(\wedge_{j=2}^{\rho}\left(\wedge_{\alpha=1}^{\delta} d x_{1 j}^{\alpha}\right)\right)+O\left(\left|x-x_{0}\right|^{2}\right)
$$

with $b$ being a constant. Since

$$
\begin{aligned}
\mathscr{L}_{u}\left(d x_{11}\right)= & d \mathscr{L}_{u}\left(x_{11}\right)=-d\left\langle u x \mid \rho e_{11}\right\rangle=-d\left\langle x \mid \rho e_{11} u\right\rangle \\
= & -\left\langle e_{11} \mid \rho e_{11} u\right\rangle d x_{11}-\sum_{2 \leq i \leq \rho}^{1 \leq \alpha \leq \delta}\left\langle e_{1 i}^{\alpha} \mid \rho e_{11} u\right\rangle d x_{1 i}^{\alpha}, \\
\mathscr{L}_{u}\left(d x_{1 j}^{\beta}\right)= & -\left\langle e_{1 j}^{\beta} \mid \rho e_{1 j}^{\beta} u\right\rangle d x_{1 j}^{\beta}-\left\langle e_{11} \mid \rho e_{1 j}^{\beta} u\right\rangle d x_{11} \\
& -\sum_{(i, \alpha) \neq(j, \beta)}\left\langle e_{1 i}^{\alpha} \mid \rho e_{1 j}^{\beta} u\right\rangle d x_{1 i}^{\alpha},
\end{aligned}
$$

we have

$$
\begin{aligned}
\left.\mathscr{L}_{u}(\operatorname{vol} \mathscr{P})\right|_{x_{0}} & =-\left.\left(\left\langle e_{11} \mid \rho e_{11} u\right\rangle+\sum_{j \geq 2,1 \leq \beta \leq \delta}\left\langle e_{1 j}^{\beta} \mid \rho e_{1 j}^{\beta} u\right\rangle\right) \operatorname{vol} \mathscr{P}\right|_{x_{0}} \\
& =-\left.\rho\left(\left\langle e_{11} \mid u\right\rangle+\frac{\delta}{2} \sum_{j \geq 2}\left\langle e_{11}+e_{j j} \mid u\right\rangle\right) \operatorname{vol}\right|_{x_{0}}
\end{aligned}
$$




$$
=-\left.\rho\left((1+(\rho / 2-1) \delta)\left\langle e_{11} \mid u\right\rangle+\frac{\delta}{2}\langle e \mid u\rangle\right) \operatorname{vol}_{\mathscr{P}}\right|_{x_{0}} .
$$

On the other hand,

$$
\left.\mathscr{L}_{u}\left(\frac{1}{r}\right)\right|_{x_{0}}=\left.\frac{\langle u \mid x\rangle}{r} \cdot \frac{1}{r}\right|_{x_{0}}=\left.\rho\left\langle u \mid e_{11}\right\rangle \cdot \frac{1}{r}\right|_{x_{0}} .
$$

Therefore,

$$
\begin{aligned}
\left.\mathscr{L}_{u}\left(\frac{1}{r} \operatorname{vol} \mathscr{P}\right)\right|_{x_{0}} & =-\left.\rho\left((\rho / 2-1) \delta\left\langle e_{11} \mid u\right\rangle+\frac{\delta}{2}\langle e \mid u\rangle\right) \frac{1}{r} \operatorname{vol} \mathscr{P}\right|_{x_{0}} \\
& =-\left.2 \lambda_{u} \frac{1}{r} \operatorname{vol} \mathscr{P}\right|_{x_{0}} .
\end{aligned}
$$

Then

$$
\begin{aligned}
\left(\tilde{L}_{u} \psi_{1}, \psi_{2}\right)+\left(\psi_{1}, \tilde{L}_{u} \psi_{2}\right) & =\int_{\mathscr{P}} \mathscr{L}_{u}\left(\overline{\psi_{1}} \psi_{2} \frac{1}{r} \operatorname{vol} \mathscr{P}\right) \\
& =\int_{\mathscr{P}} d \iota_{\hat{L}_{u}}\left(\overline{\psi_{1}} \psi_{2} \frac{1}{r} \operatorname{vol} \mathscr{P}\right)=0 .
\end{aligned}
$$

Here $\iota_{\hat{L}_{u}}$ is interior product of differential form with vector field $\hat{L}_{u}$.

\section{The Hidden Action on the KePler Cones}

Our recent investigation of the Kepler-type problems leads to the discovery of the hidden action of the conformal algebra on the Kepler cone. By turning arguments backward, we can say that it is this hidden action that is responsible for the existence of Kepler-type problems.

We begin with some generalities. For smooth manifold $M$, we use $\mathfrak{X}(M)$ to denote the Lie algebra of (smooth) vector fields on $M$ and $\mathscr{D}(M)$ to denote the algebra of smooth (real) differential operators on $M$.

Let $A$ be an associative algebra with identity over $\mathbb{R}$. We say that $A$ acts on $M$ hiddenly if there is an algebra homomorphism from $A$ into $\mathscr{D}(M) \otimes_{\mathbb{R}} \mathbb{C}$. For example, if $A=\mathbb{R}[t]$ (the polynomial algebra over $\mathbb{R}$ in single variable $t$ ), then the algebra homomorphism $A \rightarrow$ $\mathscr{D}(M) \otimes_{\mathbb{R}} \mathbb{C}$ sending $t$ to the Laplace operator on $M$, defines a hidden action of $A$ on $M$.

Let $\mathfrak{g}$ be a real Lie algebra. We say that $\mathfrak{g}$ acts on $M$ if there is a Lie algebra homomorphism from $\mathfrak{g}$ into $\mathfrak{X}(M)$; and we say that $\mathfrak{g}$ acts on $M$ hiddenly if the universal enveloping algebra of $\mathfrak{g}$ acts on $M$ hiddenly. It is clear that, if $\mathfrak{g}$ acts on $M$, then it acts on $M$ hiddenly; however, the converse may not be true. Note that, $\mathfrak{X}(M)$ acts on $M$, but $\mathscr{D}(M)$ acts on $M$ only hiddenly.

Let $V$ be a simple euclidean Jordan algebra. Since the automorphisms of $V$ leave the Kepler cone invariant, the derivation algebra, being the Lie algebra of automorphism group, acts on the Kepler cone. The recent investigation of the Kepler-type problems leads to the following fact: there is a natural hidden action of the conformal algebra on the Kepler cone which extends the action of the derivation algebra.

To introduce the hidden action, we fix an orthonormal basis $e_{\alpha}$ for $V$ and recall that $\tilde{L}_{u}=\hat{L}_{u}-\lambda_{u}$ where

$$
\lambda_{u}=\frac{(\rho / 2-1) \delta}{2} \frac{\langle u \mid x\rangle}{\langle e \mid x\rangle}+\frac{\rho \delta}{4}\langle u \mid e\rangle
$$


For $u, v \in V$, we introduce differential operators

$$
\tilde{S}_{u v}:=\left[\tilde{L}_{u}, \tilde{L}_{v}\right]+\tilde{L}_{u v}, \quad \tilde{X}_{u}:=-i\left[\tilde{L}_{u}, X\right], \quad \tilde{Y}_{v}:=-i\langle v \mid x\rangle
$$

where

$$
X=-\frac{1}{\langle e \mid x\rangle}\left(\hat{L}_{e}^{2}-((\rho-1) \delta-1) \hat{L}_{e}+A \sum_{\alpha, \beta}\left[\hat{L}_{e_{\alpha}}, \hat{L}_{e_{\beta}}\right]^{2}+B\right)
$$

with $A$ and $B$ being constants depending only on the Jordan algebra. Note that $X$ is independent of the choice of the orthonormal basis $e_{\alpha}$ and

$$
\tilde{S}_{u e}=\tilde{S}_{e u}=\tilde{L}_{u} .
$$

In view of part ii) of Lemma 5.1, $\tilde{S}_{u v}, \tilde{X}_{u}$ and $\tilde{Y}_{v}$ all descend to differential operators on the Kepler cone.

Lemma 6.1. i) There is a unique constant $A$ in Eq. 6.2), such that, as differential operator on $\mathscr{P}$,

$$
[X,\langle u \mid x\rangle]=2 \tilde{L}_{u}
$$

for any $u \in V$. In fact

$$
A^{-1}=\frac{\rho^{2}}{2}\left(1+\frac{\delta}{4}(\rho-2)\right) .
$$

ii) Let $\Delta_{\mathbb{P}}$ be the Laplace operator on $\mathbb{P}$ and $A$ be the number in Eq. (6.4). Then

$$
\Delta_{\mathbb{P}}=A \sum_{\alpha, \beta}\left[\hat{L}_{e_{\alpha}}, \hat{L}_{e_{\beta}}\right]^{2}
$$

as differential operators on on $\mathbb{P}$. Consequently,

$$
X=-\langle e \mid x\rangle \Delta_{\mathscr{P}}-\frac{B}{\langle e \mid x\rangle} .
$$

Here, $\Delta_{\mathscr{P}}$ is the Laplace operator on $\mathscr{P}$.

Proof. i) For simplicity, we write $\langle x \mid e\rangle$ as $r$. Since

$$
\begin{aligned}
{[X,\langle u \mid x\rangle] } & =-\frac{1}{r}\left[\hat{L}_{e}^{2}-((\rho-1) \delta-1) \hat{L}_{e}+A \sum_{\alpha, \beta}\left[\hat{L}_{e_{\alpha}}, \hat{L}_{e_{\beta}}\right]^{2},\langle u \mid x\rangle\right] \\
& =-\frac{1}{r}\left(-2\langle u \mid x\rangle \tilde{L}_{e}+\left[A \sum_{\alpha, \beta}\left[\hat{L}_{e_{\alpha}}, \hat{L}_{e_{\beta}}\right]^{2},\langle u \mid x\rangle\right]\right),
\end{aligned}
$$

we just need to show that

$$
\left[A \sum_{\alpha, \beta}\left[\hat{L}_{e_{\alpha}}, \hat{L}_{e_{\beta}}\right]^{2},\langle u \mid x\rangle\right]=-2 r \tilde{L}_{u}+2\langle u \mid x\rangle \tilde{L}_{e}
$$

or

$$
\left\{\begin{aligned}
A \sum_{\alpha, \beta}\left\langle\left[L_{e_{\alpha}}, L_{e_{\beta}}\right] u \mid x\right\rangle\left[\hat{L}_{e_{\alpha}}, \hat{L}_{e_{\beta}}\right] & =-r \hat{L}_{u}+\langle u \mid x\rangle \hat{L}_{e} \\
A\left\langle\sum_{\alpha, \beta}\left[L_{e_{\alpha}}, L_{e_{\beta}}\right]^{2} u \mid x\right\rangle & =-\frac{\rho \delta}{2}(\langle u \mid x\rangle-r\langle u \mid e\rangle)
\end{aligned}\right.
$$


for any $u \in V$ and any $x \in \mathscr{P}$. So it suffices to show that

$$
A \sum_{\alpha, \beta}\left|\left[L_{e_{\alpha}}, L_{e_{\beta}}\right] x\right\rangle\left\langle\left[L_{e_{\alpha}}, L_{e_{\beta}}\right] x\left|=r \sum_{\alpha}\right| e_{\alpha}\right\rangle\left\langle e_{\alpha} x|-| x\right\rangle\langle x|
$$

and

$$
A \sum_{\alpha, \beta}\left[L_{e_{\alpha}}, L_{e_{\beta}}\right]^{2} x=-\frac{\rho \delta}{2}(x-r e)
$$

for any $x \in \mathscr{P}$. Eq. 6.9 is the content of part i) of Lemma5.1 with

$$
A^{-1}=\frac{\rho^{2}}{2}\left(1+\frac{\delta}{4}(\rho-2)\right) \text {. }
$$

Eq. (6.10) is clear except that we don't know what the constant $A$ is. Here is a way to find $A$ : taking inner product with $x$, we have

$$
A \sum_{\alpha, \beta}\left\|\left[L_{e_{\alpha}}, L_{e_{\beta}}\right] x\right\|^{2}=\frac{(\rho-1) \delta}{2}\|x\|^{2} .
$$

On the other hand, by taking the trace of Eq. 6.97, we also arrive at Eq. 6.11; ; so Eq. (6.10) is a consequence of Eq. (6.9).

ii) In view of identity (6.7) and the fact that both $\Delta_{\mathbb{P}}$ and $A \sum_{\alpha, \beta}\left[\hat{L}_{e_{\alpha}}, \hat{L}_{e_{\beta}}\right]^{2}$ are 2nd order differential operators without the constant terms, it suffices to show that

$$
\left[\Delta_{\mathbb{P}},\langle u \mid x\rangle\right]=-2 r \tilde{L}_{u}+2\langle u \mid x\rangle \tilde{L}_{e} .
$$

To verify identity (6.12) at a point $x_{0} \in \mathbb{P}$, we choose a Jordan frame $\left\{e_{11}, \ldots, e_{\rho \rho}\right\}$ such that $x_{0}=\sqrt{2 \rho} e_{11}$ and write variable

$$
x=\sum_{1 \leq i \leq \rho} x_{i i} e_{i i}+\sum_{1 \leq i<j \leq \rho}^{1 \leq \alpha \leq \delta} x_{i j}^{\alpha} e_{i j}^{\alpha}
$$

where $e_{i i}$ 's, $e_{i j}^{\alpha}$ 's are mutually orthogonal with length $\frac{1}{\sqrt{\rho}}$.

By solving equation $x^{2}=\operatorname{tr} x x$ and $\operatorname{tr} x=\sqrt{2 \rho}$, we know that $x_{1 j}^{\alpha}$ 's $(j>1)$ are independent real variables and the Taylor expansion of other variables has no linear terms in $x_{1 j}^{\alpha}$ 's. Therefore, around point $x_{0}$, we have

$$
\begin{aligned}
d s_{\mathbb{P}}^{2} & =\left.d s_{E}^{2}\right|_{\mathbb{P}}=\frac{1}{\rho} \sum_{2 \leq i \leq \rho}^{1 \leq \alpha \leq \delta}\left(d x_{1 i}^{\alpha}\right)^{2}+O\left(\left|x-x_{0}\right|^{2}\right), \\
\langle u \mid x\rangle & =\left\langle u \mid x_{0}\right\rangle+\sum_{2 \leq i \leq \rho}^{1 \leq \alpha \leq \delta}\left\langle u \mid x_{1 i}^{\alpha} e_{1 i}^{\alpha}\right\rangle+O\left(\left|x-x_{0}\right|^{2}\right) .
\end{aligned}
$$

Thus

$$
\begin{aligned}
\text { LHS of Eq. (6.12) }\left.\right|_{x_{0}} & =\left.\rho \sum_{2 \leq i \leq \rho}^{1 \leq \alpha \leq \delta}\left[\frac{\partial^{2}}{\partial\left(x_{1 i}^{\alpha}\right)^{2}},\langle u \mid x\rangle\right]\right|_{x_{0}} \\
& =\left.2 \rho \sum_{2 \leq i \leq \rho}^{1 \leq \alpha \leq \delta}\left\langle u \mid e_{1 i}^{\alpha}\right\rangle \frac{\partial}{\partial x_{1 i}^{\alpha}}\right|_{x_{0}}
\end{aligned}
$$




$$
+\left.\rho \sum_{2 \leq i \leq \rho}^{1 \leq \alpha \leq \delta} \frac{\partial^{2}}{\partial\left(x_{1 i}^{\alpha}\right)^{2}}(\langle u \mid x\rangle)\right|_{x_{0}}
$$

On the other hand,

$$
\begin{aligned}
\text { RHS of Eq. [6.12) }\left.\right|_{x_{0}}= & \left.2 \rho \sum_{2 \leq i \leq \rho}^{1 \leq \alpha \leq \delta}\left\langle u \mid e_{1 i}^{\alpha}\right\rangle \frac{\partial}{\partial x_{1 i}^{\alpha}}\right|_{x_{0}} \\
& +\delta \sqrt{\rho / 2}\left(-\rho\left\langle u \mid e_{11}\right\rangle+\langle u \mid e\rangle\right) .
\end{aligned}
$$

Therefore, all need to do is to verify that

$$
\left.\sum_{2 \leq i \leq \rho}^{1 \leq \alpha \leq \delta} \frac{\partial^{2}}{\partial\left(x_{1 i}^{\alpha}\right)^{2}}(\langle u \mid x\rangle)\right|_{x_{0}}=\frac{\delta}{\sqrt{2 \rho}}\left(-\rho\left\langle u \mid e_{11}\right\rangle+\langle u \mid e\rangle\right)
$$

or

$$
\left.\sum_{2 \leq i \leq \rho}^{1 \leq \alpha \leq \delta} \frac{\partial^{2}}{\partial\left(y_{1 i}^{\alpha}\right)^{2}}(\langle u \mid y\rangle)\right|_{y=0}=\delta\left(-\rho\left\langle u \mid e_{11}\right\rangle+\langle u \mid e\rangle\right),
$$

here $y$ satisfies the following condition:

$$
2 e_{11} y+y^{2}=y, \quad \operatorname{tr} y=0,
$$

SO

$$
y=t+\rho\left[-\left\langle t^{2} \mid e_{11}\right\rangle e_{11}+\sum_{j=2}^{\rho}\left\langle t^{2} \mid e_{j j}\right\rangle e_{j j}+\sum_{2 \leq k<l \leq \rho}^{1 \leq \beta \leq \delta}\left\langle t^{2} \mid e_{k l}^{\beta}\right\rangle e_{k l}^{\beta}\right]+o\left(t^{2}\right),
$$

where $t$ is a free parameter taking values in $\oplus_{j=2}^{\rho} V_{1 j}$.

So, in view of the fact that $\left(e_{1 i}^{\alpha}\right)^{2}=\frac{1}{2}\left(e_{11}+e_{i i}\right)$, we have

$$
\begin{aligned}
\text { LHS of Eq. [6.15] }= & \left.\sum_{2 \leq i \leq \rho}^{1 \leq \alpha \leq \delta} \frac{\partial^{2}}{\partial\left(t_{1 i}^{\alpha}\right)^{2}}(\langle u \mid y\rangle)\right|_{t=0} \\
= & 2 \rho \sum_{2 \leq i \leq \rho}^{1 \leq \alpha \leq \delta}\left[-\left\langle\left(e_{1 i}^{\alpha}\right)^{2} \mid e_{11}\right\rangle\left\langle u \mid e_{11}\right\rangle\right. \\
& +\sum_{j=2}^{\rho}\left\langle\left(e_{1 i}^{\alpha}\right)^{2} \mid e_{j j}\right\rangle\left\langle u \mid e_{j j}\right\rangle \\
& \left.+\sum_{2 \leq k<1 \leq \rho}^{1 \leq \beta \leq \delta}\left\langle\left(e_{1 i}^{\alpha}\right)^{2} \mid e_{k l}^{\beta}\right\rangle\left\langle u \mid e_{k l}^{\beta}\right\rangle\right] \\
= & \sum_{2 \leq i \leq \rho}^{1 \leq \alpha \leq \delta}\left(-\left\langle u \mid e_{11}\right\rangle+\left\langle u \mid e_{i i}\right\rangle\right) \\
= & \delta \sum_{2 \leq i \leq \rho}\left(-\left\langle u \mid e_{11}\right\rangle+\left\langle u \mid e_{i i}\right\rangle\right) \\
= & \delta\left(-(\rho-1)\left\langle u \mid e_{11}\right\rangle+\left\langle u \mid e-e_{11}\right\rangle\right) \\
= & \delta\left(-\rho\left\langle u \mid e_{11}\right\rangle+\langle u \mid e\rangle\right) \\
= & \operatorname{RHS~of~Eq.~[6.15].~}
\end{aligned}
$$


6.1. Hidden Action. The following theorem implies that there is a hidden action of the conformal algebra on the Kepler cone.

Theorem 1 (Hidden Action/Dynamical Symmetry). Let $V$ be a simple euclidean Jordan algebra with rank $\rho \geq 2$ and degree $\delta$. There are unique constants $A$ and $B$ in Eq. 6.2) such that, as differential operators on the Kepler cone, $\tilde{S}_{u v}, \tilde{X}_{u}$ and $\tilde{Y}_{v}$ satisfy the TKK commutation relation (3.3) for the conformal algebra, i.e.,

$$
\begin{gathered}
{\left[\tilde{X}_{u}, \tilde{X}_{v}\right]=0, \quad\left[\tilde{Y}_{u}, \tilde{Y}_{v}\right]=0, \quad\left[\tilde{X}_{u}, \tilde{Y}_{v}\right]=-2 \tilde{S}_{u v},} \\
{\left[\tilde{S}_{u v}, \tilde{X}_{z}\right]=\tilde{X}_{\{u v z\}}, \quad\left[\tilde{S}_{u v}, \tilde{Y}_{z}\right]=-\tilde{Y}_{\{v u z\}},} \\
{\left[\tilde{S}_{u v}, \tilde{S}_{z w}\right]=\tilde{S}_{\{u v z\} w}-\tilde{S}_{z\{v u w\}}}
\end{gathered}
$$

for $u, v, z, w$ in $V$. In fact,

$$
A=\frac{2 / \rho^{2}}{1+\frac{\delta}{4}(\rho-2)}, \quad B=\frac{\delta}{8}(\rho-2)\left(\left(\frac{3 \rho}{2}-1\right) \delta-2\right) .
$$

Remark 6.1. Here are the more explicit values of $A$ and $B$ :

\begin{tabular}{|c|c|c|c|c|c|}
\hline$V$ & $\Gamma(n)$ & $\mathcal{H}_{n}(\mathbb{R})$ & $\mathcal{H}_{n}(\mathbb{C})$ & $\mathcal{H}_{n}(\mathbb{H})$ & $\mathcal{H}_{3}(\mathbb{O})$ \\
\hline$A$ & $\frac{1}{2}$ & $\frac{8}{n^{2}(n+2)}$ & $\frac{4}{n^{3}}$ & $\frac{2}{n^{2}(n-1)}$ & $\frac{2}{27}$ \\
$B$ & 0 & $\frac{3(n-2)^{2}}{16}$ & $\frac{(n-2)(3 n-4)}{4}$ & $3(n-1)(n-2)$ & 26 \\
\hline
\end{tabular}

In the case $\rho=1$, a similar theorem is valid except that $A=0$ and $B$ is not unique.

Proof. For any function $f$ on $V$ and $u, v \in V$, we have

$$
\left(\tilde{Y}_{u} \tilde{Y}_{v}\right)(f)(x)=-\langle u \mid x\rangle\langle v \mid x\rangle f(x),
$$

so $\left[\tilde{Y}_{u}, \tilde{Y}_{v}\right]=0$ for any $u, v \in V$. The rest of the proof is divided into four steps.

Step one: Verify that $\left[\tilde{S}_{u v}, \tilde{Y}_{z}\right]=-\tilde{Y}_{\{v u z\}}$.

This is a simple computation:

$$
\begin{aligned}
{\left[\tilde{S}_{u v}, \tilde{Y}_{z}\right] } & =\left[\hat{S}_{u v},-i\langle z \mid x\rangle\right]=i\left\langle z \mid S_{u v}(x)\right\rangle \\
& =i\left\langle S_{v u}(z) \mid x\right\rangle=i\langle\{v u z\} \mid x\rangle \\
& =-\tilde{Y}_{\{v u z\}} .
\end{aligned}
$$

Step two: Verify that

$$
\left[\tilde{S}_{u v}, \tilde{S}_{z w}\right]=\tilde{S}_{\{u v z\} w}-\tilde{S}_{z\{v u w\}} .
$$

It is easy to see that $\tilde{S}_{u v}=\hat{S}_{u v}-\lambda_{u v}$. Thanks to Proposition 3.1. $\left[\hat{S}_{u v}, \hat{S}_{z w}\right]=$ $\hat{S}_{\{u v z\} w}-\hat{S}_{z\{v u w\}}$, so all we need to check is that

$$
\lambda_{\{u v z\} w}-\lambda_{z\{v u w\}}=\left[\hat{S}_{u v}, \lambda_{z w}\right]-\left[\hat{S}_{z w}, \lambda_{u v}\right],
$$

i.e.,

$$
\lambda_{\{u v z\} w}-\lambda_{z\{v u w\}}=-\lambda_{\{v u(z w)\}}+\lambda_{\{w z(u v)\}} .
$$


Since $\lambda_{u}$ is linear in $u$, the last equality is implied by identity

$$
L_{\{u v z\}}-L_{z} S_{v u}=-S_{v u} L_{z}+S_{(u v) z} \text {, i.e., } L_{\{u v z\}}=S_{(z v) u}-S_{v(z u)}+S_{(u v) z}
$$

or equivalently

$$
0=\left[L_{z v}, L_{u}\right]-\left[L_{v}, L_{z u}\right]+\left[L_{u v}, L_{z}\right]
$$

— the polarization of identity $\left[L_{u^{2}}, L_{u}\right]=0$.

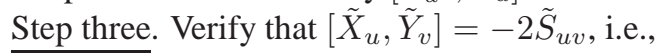

$$
\left[\left[\tilde{L}_{u}, X\right],\langle v \mid x\rangle\right]=2 \tilde{S}_{u v} .
$$

This is a consequence of part i) of Lemma 6.1

$$
\begin{aligned}
{\left[\left[\tilde{L}_{u}, X\right],\langle x \mid v\rangle\right] } & =\left[[\langle x \mid v\rangle, X], \tilde{L}_{u}\right]+\left[\left[\tilde{L}_{u},\langle x \mid v\rangle\right], X\right] \\
& =\left[-2 \tilde{L}_{v}, \tilde{L}_{u}\right]+[-\langle x \mid u v\rangle, X] \\
& =\left[-2 \tilde{L}_{v}, \tilde{L}_{u}\right]+2 \tilde{L}_{u v}=2 \tilde{S}_{u v} .
\end{aligned}
$$

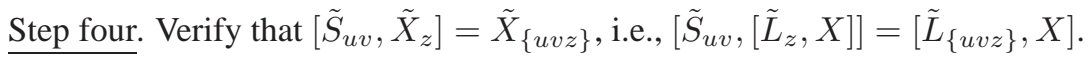

Note that $X$ is invariant under $\mathfrak{d e r}$ and $\tilde{S}_{e u}=\tilde{S}_{u e}=\tilde{L}_{u}$, in view of Eq. 6.17), we have

$$
\begin{aligned}
{\left[\tilde{S}_{u v},\left[\tilde{L}_{z}, X\right]\right] } & =\left[X,\left[\tilde{L}_{z}, \tilde{S}_{u v}\right]\right]+\left[\tilde{L}_{z},\left[\tilde{S}_{u v}, X\right]\right] \\
& =\left[\tilde{L}_{\{u v z\}}-\tilde{S}_{z(v u)}, X\right]+\left[\tilde{L}_{z},\left[\tilde{S}_{u v}, X\right]\right] \\
& =\left[\tilde{L}_{\{u v z\}}-\tilde{L}_{z(v u)}, X\right]+\left[\tilde{L}_{z},\left[\tilde{L}_{u v}, X\right]\right] \\
& =\left[\tilde{L}_{\{u v z\}}, X\right]-\left[\tilde{L}_{z(u v)}, X\right]+\left[\tilde{L}_{z},\left[\tilde{L}_{u v}, X\right]\right] .
\end{aligned}
$$

So it suffices to show that

$$
\left[\tilde{L}_{u v}, X\right]=\left[\tilde{L}_{u},\left[\tilde{L}_{v}, X\right]\right]
$$

for any $u, v \in V$. To prove it, we let

$$
O=\left[\tilde{L}_{u v}, X\right]-\left[\tilde{L}_{u},\left[\tilde{L}_{v}, X\right]\right]
$$

and show that, 1) for any $z \in V, O_{z}:=[O,\langle x \mid z\rangle]=0$, 2) $O(1)=0$. Eq. (6.18) implies that

$$
\begin{aligned}
{\left[\left[\tilde{L}_{u},\left[\tilde{L}_{v}, X\right]\right],\langle x \mid z\rangle\right] } & =\left[\left[\langle x \mid z\rangle,\left[\tilde{L}_{v}, X\right]\right], \tilde{L}_{u}\right]+\left[\left[\tilde{L}_{u},\langle x \mid z\rangle\right],\left[\tilde{L}_{v}, X\right]\right] \\
& =\left[\left[\langle x \mid z\rangle,\left[\tilde{L}_{v}, X\right]\right], \tilde{L}_{u}\right]+\left[-\langle x \mid u z\rangle,\left[\tilde{L}_{v}, X\right]\right] \\
& =\left[-2 \tilde{S}_{v z}, \tilde{L}_{u}\right]+2 \tilde{S}_{v(u z)} .
\end{aligned}
$$

So, in view of Eq. 6.17, we have

$$
\begin{aligned}
O_{z} / 2 & =\tilde{S}_{(u v) z}+\left[\tilde{S}_{v z}, \tilde{L}_{u}\right]-\tilde{S}_{v(u z)} \\
& =\tilde{S}_{(u v) z}-\tilde{S}_{v(u z)}-\left[\tilde{S}_{u e}, \tilde{S}_{v z}\right] \\
& =0
\end{aligned}
$$

The proof of $O(1)=0$ is a long computation, so its details are provided in the appendix.

Step five. Verify that $\left[\tilde{X}_{u}, \tilde{X}_{v}\right]=0$, i.e., $\left[\left[\tilde{L}_{u}, X\right],\left[\tilde{L}_{v}, X\right]\right]=0$.

Eq. (6.19) implies that

$$
\begin{aligned}
{\left[\left[\tilde{L}_{u}, X\right],\left[\tilde{L}_{v}, X\right]\right] } & \left.=\left[\left[\tilde{L}_{u},\left[\tilde{L}_{v}, X\right]\right], X\right]+\left[\left[\tilde{L}_{v}, X\right], X\right], \tilde{L}_{u}\right] \\
& =\left[\left[\tilde{L}_{u v}, X\right], X\right]+\left[\left[\left[\tilde{L}_{v}, X\right], X\right], \tilde{L}_{u}\right] .
\end{aligned}
$$

So it suffices to show that

$$
\left[\left[\tilde{L}_{u}, X\right], X\right]=0
$$


for any $u \in V$. To prove Eq. [6.21), as in step four, we let $\mathscr{O}=\left[\left[\tilde{L}_{u}, X\right], X\right]$, and show that, 1) for any $\left.z \in V, \mathscr{O}_{z}:=[\mathscr{O},\langle x \mid z\rangle]=0,2\right) \mathscr{O}(1)=0$.

In view of Eqs. 6.19) and (6.18), part i) of Lemma 6.1 and the fact that $X$ is invariant under the action of $\mathfrak{d e r}$, we have

$$
\begin{aligned}
{[\mathscr{O},\langle x \mid z\rangle] } & =\left[[\langle x \mid z\rangle, X],\left[\tilde{L}_{u}, X\right]\right]+\left[\left[\left[\tilde{L}_{u}, X\right],\langle x \mid z\rangle\right], X\right] \\
& =-2\left[\tilde{L}_{z},\left[\tilde{L}_{u}, X\right]\right]+\left[2 \tilde{S}_{u z}, X\right] \\
& =-2\left[\tilde{L}_{z},\left[\tilde{L}_{u}, X\right]\right]+\left[2 \tilde{L}_{u z}, X\right]=0 .
\end{aligned}
$$

The proof of $\mathscr{O}(1)=0$ is a long computation, so its details are provided in the appendix.

6.2. Quadratic Relations for the Hidden Action. Let $V_{0}$ be the orthogonal complement of $e$ in $V, D=\operatorname{dim} V_{0}$ and $\left\{e_{\alpha}\right\}_{1 \leq \alpha \leq D}$ be the orthonormal basis for $V_{0}$. We write $e$ as $e_{0}$ and $\left\langle x \mid e_{\alpha}\right\rangle$ as $x_{\alpha}$. Note that $\tilde{S}_{e u}=\tilde{S}_{u e}=\tilde{L}_{u}$.

Theorem 2. (The Primary Quadratic Relation)

$$
\frac{2}{\rho} \sum_{0 \leq \alpha \leq D} \tilde{L}_{e_{\alpha}}^{2}-\tilde{L}_{e}^{2}-\frac{1}{2}\left\{\tilde{X}_{e}, \tilde{Y}_{e}\right\}=-a
$$

Here, $a=\frac{\rho \delta}{4}\left(1+\frac{(\rho-2) \delta}{4}\right)$.

Proof. Let $\mathscr{O}$ be the left hand side of Eq. 6.23). We just need to show that, on the Kepler cone we have 1) $\left[\mathscr{O}, \tilde{Y}_{u}\right]=0$ and 2) $\mathscr{O}(1)=-a$.

Since

$$
\begin{aligned}
{\left[\tilde{L}_{e_{\alpha}}^{2}, \tilde{Y}_{u}\right] } & =\left\{\tilde{L}_{e_{\alpha}},\left[\tilde{L}_{e_{\alpha}}, \tilde{Y}_{u}\right]\right\}=\left\{\tilde{L}_{e_{\alpha}},-\tilde{Y}_{u e_{\alpha}}\right\} \\
& =-2 \tilde{Y}_{u e_{\alpha}} \tilde{L}_{e_{\alpha}}-\left[\tilde{L}_{e_{\alpha}}, \tilde{Y}_{u e_{\alpha}}\right] \\
& =-2 \tilde{Y}_{u e_{\alpha}} \tilde{L}_{e_{\alpha}}+\tilde{Y}_{\left(u e_{\alpha}\right) e_{\alpha}}
\end{aligned}
$$

we have

$$
\begin{aligned}
\sum_{0 \leq \alpha \leq D}\left[\tilde{L}_{e_{\alpha}}^{2}, \tilde{Y}_{u}\right] & =\sum_{0 \leq \alpha \leq D}-2 \tilde{Y}_{u e_{\alpha}}\left(\hat{L}_{e_{\alpha}}-\lambda_{e_{\alpha}}\right)+\tilde{Y}_{\left(u e_{\alpha}\right) e_{\alpha}} \\
& =2 i \hat{L}_{u x}-2 i \lambda_{u x}+\rho\left(1+\frac{\rho-2}{4} \delta\right) \tilde{Y}_{u}+\frac{\rho \delta}{4} \operatorname{tr} u \tilde{Y}_{e} \\
& =2 i \hat{L}_{u x}+\rho\left(1+\frac{3 \rho-4}{4} \delta\right) \tilde{Y}_{u}+\frac{\rho \delta}{4} \operatorname{tr} u \tilde{Y}_{e},
\end{aligned}
$$

here, we have used the identity

$$
\sum_{\alpha} L_{e_{\alpha}}^{2}=\rho\left(1+\frac{\rho-2}{4} \delta\right) L_{e}+\frac{\rho^{2} \delta}{4}|e\rangle\langle e| .
$$

On the other hand,

$$
\begin{aligned}
{\left[\tilde{L}_{e}^{2}+\frac{1}{2}\left\{\tilde{X}_{e}, \tilde{Y}_{e}\right\}, \tilde{Y}_{u}\right] } & =\left\{\tilde{L}_{e},\left[\tilde{L}_{e}, \tilde{Y}_{u}\right]\right\}+\frac{1}{2}\left\{\left[\tilde{X}_{e}, \tilde{Y}_{u}\right], \tilde{Y}_{e}\right\} \\
& =-2\left(\tilde{Y}_{u} \tilde{L}_{e}+\tilde{Y}_{e} \tilde{L}_{u}-\tilde{Y}_{u}\right),
\end{aligned}
$$

so we have

$$
\begin{aligned}
{\left[\mathscr{O}, \tilde{Y}_{u}\right]=} & \frac{4 i}{\rho} \hat{L}_{u x}+2\left(1+\frac{3 \rho-4}{4} \delta\right) \tilde{Y}_{u}+\frac{\delta}{2} \operatorname{tr} u \tilde{Y}_{e} \\
& +2\left(\tilde{Y}_{u} \hat{L}_{e}+\tilde{Y}_{e} \hat{L}_{u}-\tilde{Y}_{u}\right)-2\left(\tilde{Y}_{u} \lambda_{e}+\tilde{Y}_{e} \lambda_{u}\right)
\end{aligned}
$$




$$
\begin{aligned}
& =\frac{4 i}{\rho} \hat{L}_{u x}+2\left(\tilde{Y}_{u} \hat{L}_{e}+\tilde{Y}_{e} \hat{L}_{u}\right) \\
& =\frac{2 i}{\rho} \sum_{0 \leq \beta \leq D} \hat{L}_{u}\left(\left\langle x^{2}-\operatorname{tr} x x \mid \not \partial\right\rangle\right) \\
& =0
\end{aligned}
$$

as differential operator on the Kepler cone.

Since

$$
\begin{aligned}
\sum_{0 \leq \alpha \leq D} \tilde{L}_{e_{\alpha}}^{2}(1) & =\sum_{\substack{0 \leq \alpha \leq D \\
0 \leq D}}-\hat{L}_{e_{\alpha}}\left(\lambda_{e_{\alpha}}\right)+\lambda_{e_{\alpha}}^{2} \\
& =\frac{\rho(\rho-2)}{4} \delta \lambda_{e}+\frac{\rho^{2}}{8} \delta \lambda_{e}=\frac{\rho(3 \rho / 2-2)}{4} \delta \lambda_{e},
\end{aligned}
$$

and

$$
-\tilde{L}_{e}^{2}(1)-\frac{1}{2}\left\{\tilde{X}_{e}, \tilde{Y}_{e}\right\}(1)=-\lambda_{e}^{2}-\lambda_{e}-B,
$$

we have

$$
\begin{aligned}
-a & =\frac{(3 \rho / 2-2)}{2} \delta \lambda_{e}-\lambda_{e}^{2}-\lambda_{e}-B \\
& =-\frac{\rho \delta}{4}\left(1+\frac{(\rho-2) \delta}{4}\right),
\end{aligned}
$$

i.e.,

$$
a=\frac{\rho \delta}{4}\left(1+\frac{(\rho-2) \delta}{4}\right) .
$$

Corollary 1. (The Secondary Quadratic Relations)

$$
\begin{array}{r}
\sum_{0 \leq \alpha \leq D}\left\{\tilde{X}_{e_{\alpha}}, \tilde{L}_{e_{\alpha}}\right\}=\rho\left\{\tilde{X}_{e}, \tilde{L}_{e}\right\}, \quad \sum_{0 \leq \alpha \leq D}\left\{\tilde{Y}_{e_{\alpha}}, \tilde{L}_{e_{\alpha}}\right\}=\rho\left\{\tilde{Y}_{e}, \tilde{L}_{e}\right\}, \\
\sum_{0 \leq \alpha \leq D} \tilde{X}_{e_{\alpha}}^{2}=\rho \tilde{X}_{e}^{2}, \quad \sum_{0 \leq \alpha \leq D} \tilde{Y}_{e_{\alpha}}^{2}=\rho \tilde{Y}_{e}^{2}, \quad \frac{1}{2} \sum_{0 \leq \alpha \leq D}\left\{\tilde{X}_{e_{\alpha}}, \tilde{Y}_{e_{\alpha}}\right\}=\rho\left(\tilde{L}_{e}^{2}+a\right), \\
\frac{2}{\rho} \sum_{1 \leq \alpha \leq D}\left\{\tilde{L}_{e_{\alpha}, u}, \tilde{L}_{e_{\alpha}}\right\}=\frac{1}{2}\left(-\left\{\tilde{X}_{u}, \tilde{Y}_{e}\right\}+\left\{\tilde{X}_{e}, \tilde{Y}_{u}\right\}\right), \\
\quad \frac{2}{\rho} \sum_{1 \leq \alpha \leq D}\left\{\tilde{L}_{e_{\alpha}, u}, \tilde{X}_{e_{\alpha}}\right\}=-\left\{\tilde{X}_{u}, \tilde{L}_{e}\right\}+\left\{\tilde{L}_{u}, \tilde{X}_{e}\right\}, \\
\quad \sum_{1 \leq \alpha, \beta \leq D}\left[\sum_{1 \leq \alpha \leq D}\left\{\tilde{L}_{e_{\alpha}, u}, \tilde{L}_{e_{\beta}}\right]^{2}=\frac{1}{2}\left\{\tilde{X}_{e}, \tilde{Y}_{e}\right\}-\left\{\tilde{Y}_{u}, \tilde{L}_{e}\right\}-\left\{\frac{\rho \delta}{4}\left(\frac{\rho \delta}{4}-1\right) .\right.\right.
\end{array}
$$

Here, $A=\frac{2 / \rho^{2}}{1+\frac{(\rho-2) \delta}{4}}$.

Proof. The two identities

$$
\sum_{0 \leq \alpha \leq D}\left\{\tilde{X}_{e_{\alpha}}, \tilde{L}_{e_{\alpha}}\right\}=\rho\left\{\tilde{X}_{e}, \tilde{L}_{e}\right\}, \quad \sum_{0 \leq \alpha \leq D}\left\{\tilde{Y}_{e_{\alpha}}, \tilde{L}_{e_{\alpha}}\right\}=\rho\left\{\tilde{Y}_{e}, \tilde{L}_{e}\right\}
$$


can be obtained by taking the commutator of Eq. (6.23) with $\tilde{X}_{e}$ and $\tilde{Y}_{e}$ respectively. Identity

$$
\frac{2}{\rho} \sum_{1 \leq \alpha \leq D}\left\{\tilde{L}_{e_{\alpha}, e_{\beta}}, \tilde{L}_{e_{\alpha}}\right\}+\frac{1}{2}\left(\left\{\tilde{X}_{e_{\beta}}, \tilde{Y}_{e}\right\}-\left\{\tilde{X}_{e}, \tilde{Y}_{e_{\beta}}\right\}\right)=0
$$

can be obtained by forming the commutator of the identity in Eq. (6.23) with $\tilde{L}_{e_{\beta}}$.

Identities

$$
\left\{\begin{aligned}
\sum_{0 \leq \alpha \leq D} \tilde{X}_{e_{\alpha}}^{2} & =\rho \tilde{X}_{e}^{2}, \\
\sum_{0 \leq \alpha \leq D} \tilde{Y}_{e_{\alpha}}^{2} & =\rho \tilde{Y}_{e}^{2}, \\
\frac{2}{\rho} \sum_{0 \leq \alpha \leq D}\left\{\tilde{X}_{e_{\alpha}}, \tilde{Y}_{e_{\alpha}}\right\} & =4\left(\tilde{L}_{e}^{2}+a\right)
\end{aligned}\right.
$$

can be proved this way: The 1st identity here is obtained from forming the commutator of the 1st identity in Eq. 6.24) with $\tilde{X}_{e}$, and the 2nd identity here is obtained from forming the commutator of the 2nd identity in Eq. (6.24) with $\tilde{Y}_{e}$. The third identity here is obtained by first forming the commutator of the 1st identity in Eq. (6.24) with $\tilde{Y}_{e}$ and then using Eq. 6.23).

Identities

$$
\left\{\begin{array}{c}
\frac{2}{\rho} \sum_{1 \leq \alpha \leq D}\left\{\tilde{L}_{e_{\alpha}, e_{\beta}}, \tilde{X}_{e_{\alpha}}\right\}+\left\{\tilde{X}_{e_{\beta}}, \tilde{L}_{e}\right\}-\left\{\tilde{L}_{e_{\beta}}, \tilde{X}_{e}\right\}=0 \\
\frac{2}{\rho} \sum_{1 \leq \alpha \leq D}\left\{\tilde{L}_{e_{\alpha}, e_{\beta}}, \tilde{Y}_{e_{\alpha}}\right\}-\left\{\tilde{Y}_{e_{\beta}}, \tilde{L}_{e}\right\}+\left\{\tilde{L}_{e_{\beta}}, \tilde{Y}_{e}\right\}=0
\end{array}\right.
$$

can be obtained by taking the commutator of Eq. 6.25) with $\tilde{X}_{e}$ and $\tilde{Y}_{e}$ respectively.

The last identity in the corollary is a direct consequence of the definition of $X$, but can be verified to be a consequence of the TKK commutation relations in Theorem 1 and Eq. (6.23).

\section{J-KePler Problems}

Definition 2 (J-Kepler Problem). The $\mathbf{J}$-Kepler problem associated to a simple euclidean Jordan algebra with rank $\rho$ and degree $\delta$ is the quantum mechanical system for which the configuration space is the Kepler cone, and the hamiltonian is

$$
\hat{h}=-\frac{1}{2} \Delta-\left(\frac{B}{2\langle e \mid x\rangle^{2}}+\frac{1}{\langle e \mid x\rangle}\right) .
$$

Here, $\Delta$ is the (non-positive) Laplace operator on the Kepler cone, and

$$
B=\frac{\delta(\rho-2)}{8}\left(\left(\frac{3 \rho}{2}-1\right) \delta-2\right) .
$$

Remark 7.1. In view of Eq. (6.6), we have another expression for $\hat{h}$ :

$$
\hat{h}=\frac{1}{\langle e \mid x\rangle}\left(\frac{i}{2} \tilde{X}_{e}-1\right) .
$$

Remark 7.2. The J-Kepler problem is really the quantum mechanical system for which the configuration space is the geometric open cone over the projective space, and the hamiltonian is

$$
\hat{h}=-\frac{1}{2} \Delta-\left(\frac{B}{2 r^{2}}+\frac{1}{r}\right),
$$


where $\Delta$ is the (non-positive) Laplace operator on the open geometric cone over the projective space.

Remark 7.3. The classical J-Kepler problem is the classical mechanical system for which the configuration space is the Kepler cone, and the Lagrangian is

$$
L(x, \dot{x})=\frac{1}{2}|\dot{x}|^{2}+\frac{1}{\langle e \mid x\rangle} .
$$

We are now ready to state

Proposition 7.1. The J-Kepler problems are equivalent to the various Kepler-type problems constructed and analyzed in Ref. [2], but with zero magnetic charge. Here is the precise identification:

\begin{tabular}{|c|c|c|c|c|c|}
\hline$V$ & $\Gamma(n)$ & $\mathcal{H}_{n}(\mathbb{R})$ & $\mathcal{H}_{n}(\mathbb{C})$ & $\mathcal{H}_{n}(\mathbb{H})$ & $\mathcal{H}_{3}(\mathbb{O})$ \\
\hline $\begin{array}{c}\text { Kepler } \\
\text { problem }\end{array}$ & $\begin{array}{c}\text { MICZ in } \\
\text { dim. } n\end{array}$ & $\begin{array}{c}\mathrm{O}(1) \text { in } \\
\text { dim. } n\end{array}$ & $\begin{array}{c}\mathrm{U}(1) \text { in } \\
\text { dim. }(2 n-1)\end{array}$ & $\begin{array}{c}\mathrm{Sp}(1) \text { in } \\
\text { dim. }(4 n-3)\end{array}$ & exceptional \\
\hline
\end{tabular}

Proof. The proposition is clear for the MICZ-Kepler problems and the exceptional Kepler problem. For the remaining cases, all one needs is to make a transformation similar to the one appeared in the proof of Proposition 2.2 of the first paper in Ref. [2]. For example, for the $\mathrm{O}(1)$-Kepler problem in dimension $n$ with zero magnetic charge, the configuration space is $\widetilde{\mathbb{R} P^{n}}=\mathbb{R}_{*}^{n} / Z \sim-Z$ and the hamiltonian is

$$
H=-\frac{1}{8 z} \Delta_{\widetilde{R P}} \frac{1}{z}-\frac{1}{z^{2}},
$$

where $\Delta_{\widetilde{\mathbb{R} P^{n}}}$ is the Laplace operator on $\widetilde{\mathbb{R} P^{n}}$ and $z=|Z|$. Note that, with the quotient metric induced from the euclidean metric of $\mathbb{R}^{n}, \widetilde{\mathbb{R} P^{n}}$ is isometric to

$$
\left(\mathbb{R}_{+} \times \mathbb{R} P^{n-1}, d z^{2}+z^{2} d s_{F S}^{2}\right),
$$

where $d s_{F S}^{2}$ is the Fubini-Study metric on $\mathbb{R} P^{n-1}$.

To see the equivalence of the J-Kepler problem associated with $\mathcal{H}_{n}(\mathbb{R})$ with the $\mathrm{O}(1)$ Kepler problem in dimension $n$ with zero magnetic charge, we start with diffeomorphism

$$
\begin{aligned}
\widetilde{\mathbb{R P}^{n}} & \rightarrow \mathscr{P} \\
{[Z] } & \mapsto n Z Z^{\prime}
\end{aligned}
$$

or equivalently diffeomorphism $\pi$ :

$$
\begin{aligned}
\widetilde{\mathbb{R} P^{n}} & \rightarrow \mathbb{R}_{+} \times \mathbb{P} \\
{[Z] } & \mapsto\left(z^{2}, \sqrt{2 n} \frac{Z Z^{\prime}}{z^{2}}\right) .
\end{aligned}
$$

Here $Z$ is viewed as a column vector in $\mathbb{R}^{n}$ and $Z^{\prime}$ is the transpose of $Z$.

Under $\pi$, we have

$$
\pi^{*}\left(d r^{2}+r^{2} d s_{\mathbb{P}}^{2}\right)=(2 z)^{2}\left(d z^{2}+z^{2} d s_{F S}^{2}\right),
$$

i.e.,

$$
\pi^{*}\left(d s_{\mathscr{P}}^{2}\right)=(2 z)^{2} d s_{\widetilde{\mathbb{R} P^{n}}}^{2}, \quad \text { so } \quad \pi^{*}\left(\operatorname{vol}_{\mathscr{P}}\right)=(2 z)^{n} \operatorname{vol}_{\widetilde{\mathbb{R} P^{n}}} .
$$

Let $\Psi_{i}(i=1$ or 2$)$ be a wave-function for the J-Kepler problem associated with $\mathcal{H}_{n}(\mathbb{R})$, and

$$
\psi_{i}(z, \Theta):=(2 z)^{\frac{n}{2}} \pi^{*}\left(\Psi_{i}\right)(z, \Theta) .
$$


Then it is not hard to see that

$$
\int_{\widetilde{R} P^{n}} \overline{\psi_{1}} \psi_{2} \operatorname{vol}_{\widetilde{\mathbb{R} P^{n}}}=\int_{\widetilde{\mathbb{R} P^{n}}} \overline{\pi^{*}\left(\Psi_{1}\right)} \pi^{*}\left(\Psi_{2}\right) \pi^{*}(\operatorname{vol} \mathscr{P})=\int_{\mathscr{P}} \overline{\Psi_{1}} \Psi_{2} \operatorname{vol} \mathscr{P}
$$

and

$$
\begin{aligned}
& \int_{\widetilde{\mathbb{R P n}}} \overline{\psi_{1}} H \psi_{2} \operatorname{vol}_{\widetilde{R P^{n}}}=\int_{\widetilde{\mathbb{R P n}}} \overline{\pi^{*}\left(\Psi_{1}\right)} \frac{1}{z^{\frac{n}{2}}} H z^{\frac{n}{2}} \pi^{*}\left(\Psi_{2}\right) \pi^{*}(\operatorname{vol} \mathscr{P}) \\
& =\int_{\mathscr{P}} \overline{\Psi_{1}} \frac{1}{z^{\frac{n}{2}}} H z^{\frac{n}{2}} \Psi_{2} \operatorname{vol}_{\mathscr{P}} \text {. }
\end{aligned}
$$

Since

$$
\begin{aligned}
\frac{1}{z^{\frac{n}{2}}} H z^{\frac{n}{2}} & =-\frac{1}{8 z^{\frac{n}{2}+1}} \Delta_{\widetilde{\mathbb{R P}}} z^{\frac{n}{2}-1}-\frac{1}{z^{2}} \\
& =-\frac{1}{8 z^{\frac{n}{2}+1}}\left(\frac{1}{z^{n-1}} \partial_{z} z^{n-1} \partial_{z}+\frac{1}{z^{2}} \Delta_{F S}\right) z^{\frac{n}{2}-1}-\frac{1}{z^{2}} \\
& =-\frac{1}{8 z^{\frac{3 n}{2}}} \partial_{z} z^{n-1} \partial_{z} z^{\frac{n}{2}-1}-\frac{1}{8 z^{4}} \Delta_{F S}-\frac{1}{z^{2}} \\
& =-\frac{1}{2 r^{\frac{3 n}{4}-\frac{1}{2}}} \partial_{r} r^{\frac{n}{2}} \partial_{r} r^{\frac{n}{4}-\frac{1}{2}}-\frac{1}{2 r^{2}} \Delta_{\mathbb{P}}-\frac{1}{r} \\
& =-\frac{1}{2}\left(\partial_{r}^{2}+\frac{n-1}{r} \partial_{r}+\frac{\left(\frac{n}{4}-\frac{1}{2}\right)\left(\frac{3 n}{4}-\frac{3}{2}\right)}{r^{2}}+\frac{1}{r^{2}} \Delta_{\mathbb{P}}\right)-\frac{1}{r} \\
& =-\frac{1}{2} \Delta-\frac{3(n-2)^{2}}{32 r^{2}}-\frac{1}{r}=\hat{h},
\end{aligned}
$$

we have the equivalence of the J-Kepler problem associated with $\mathcal{H}_{n}(\mathbb{R})$ with the $\mathrm{O}(1)$ Kepler problem in dimension $n$ with zero magnetic charge.

7.1. The Lenz Vector. The Lenz vector exists for J-Kepler problems.

Definition 3 (Lenz vector). The Lenz vector for the J-Kepler problem is

$$
A_{u}:=\frac{1}{\langle e \mid x\rangle}\left[\tilde{L}_{u},(\langle e \mid x\rangle)^{2} \hat{h}\right]
$$

i.e.,

$$
A_{u}=\frac{i}{2} \tilde{X}_{u}-\langle u \mid x\rangle \hat{h}=\frac{i}{2}\left(\tilde{X}_{u}-\frac{\langle u \mid x\rangle}{\langle e \mid x\rangle} \tilde{X}_{e}\right)+\frac{\langle u \mid x\rangle}{\langle e \mid x\rangle}
$$

Note that $A_{e}=1$. One might call $\vec{A}:=\left(A_{e_{1}}, \ldots, A_{e_{D}}\right)$ the Lenz vector, that is because $\vec{A}$ is precisely the usual Lenz vector when the Jordan algebra is the Minkowski space.

Theorem 3. For $u, v \in V$, we let $L_{u, v}:=\left[\hat{L}_{u}, \hat{L}_{v}\right]$. Then we have commutation relations:

$$
\begin{array}{|ll|}
{\left[L_{u, v}, \hat{h}\right]} & =0 \\
{\left[L_{u, v}, L_{z, w}\right]} & =L_{\left[L_{u}, L_{v}\right] z, w}+L_{z,\left[L_{u}, L_{v}\right] w} \\
{\left[L_{u, v}, A_{z}\right]} & =A_{\left[L_{u}, L_{v}\right] z} \\
{\left[A_{u}, \hat{h}\right]} & =0 \\
{\left[A_{u}, A_{v}\right]} & =-2 \hat{h} L_{u, v} .
\end{array}
$$

Remark 7.4. 1) $L_{u, v}=\left[\tilde{L}_{u}, \tilde{L}_{v}\right]$. 2) $A_{u}, \hat{h}$ and $i L_{u, v}$ are all hermitian operators with respect to inner product

$$
(\psi, \phi) \mapsto \int_{\mathscr{P}} \psi^{*} \phi \operatorname{vol} \mathscr{P}
$$


Proof. Theorem 1 implies that

$$
\left[L_{u, v}, \tilde{X}_{z}\right]=\tilde{X}_{\left[L_{u}, L_{v}\right] z}, \quad\left[L_{u, v}, \tilde{Y}_{z}\right]=\tilde{Y}_{\left[L_{u}, L_{v}\right] z}, \quad\left[L_{u, v}, \tilde{L}_{z}\right]=\tilde{L}_{\left[L_{u}, L_{v}\right] z} .
$$

In particular, we have $\left[L_{u, v}, \tilde{X}_{e}\right]=\left[L_{u, v}, \tilde{Y}_{e}\right]=0$. Therefore, $\left[L_{u, v}, H\right]=0$,

$$
\begin{aligned}
{\left[L_{u, v}, A_{z}\right] } & =\frac{1}{\langle e \mid x\rangle}\left[L_{u, v},\left[\tilde{L}_{z},(\langle e \mid x\rangle)^{2} \hat{h}\right]\right] \\
& =\frac{1}{\langle e \mid x\rangle}\left(\left[(\langle e \mid x\rangle)^{2} \hat{h},\left[\tilde{L}_{z}, L_{u, v}\right]\right]+\left[\tilde{L}_{z},\left[L_{u, v},(\langle e \mid x\rangle)^{2} \hat{h}\right]\right]\right) \\
& =\frac{1}{\langle e \mid x\rangle}\left[(\langle e \mid x\rangle)^{2} \hat{h},-\tilde{L}_{\left[L_{u}, L_{v}\right] z}\right] \\
& =A_{\left[L_{u}, L_{v}\right] z},
\end{aligned}
$$

and

$$
\begin{aligned}
{\left[L_{u, v}, L_{z, w}\right] } & =\left[L_{u, v},\left[\tilde{L}_{z}, \tilde{L}_{w}\right]\right] \\
& =\left[\left[L_{u, v}, \tilde{L}_{z}\right], \tilde{L}_{w}\right]+\left[\tilde{L}_{z},\left[L_{u, v}, \tilde{L}_{w}\right]\right] \\
& =\left[\tilde{L}_{\left[L_{u}, L_{v}\right] z}, \tilde{L}_{w}\right]+\left[\tilde{L}_{z}, \tilde{L}_{\left.\left[L_{u}, L_{v}\right] w\right]}\right] \\
& =L_{\left[L_{u}, L_{v}\right] z, w}+L_{z,\left[L_{u}, L_{v}\right] w .}
\end{aligned}
$$

Since $\hat{h}=\frac{1}{\langle e \mid x\rangle}\left(\frac{i}{2} \tilde{X}_{e}-1\right)$, we have

$$
\begin{aligned}
{\left[A_{u}, \hat{h}\right]=} & {\left[A_{u}, \frac{1}{\langle e \mid x\rangle}\right]\left(\frac{i}{2} \tilde{X}_{e}-1\right)+\frac{1}{\langle e \mid x\rangle}\left[A_{u}, \frac{i}{2} \tilde{X}_{e}\right] } \\
= & -\frac{1}{\langle e \mid x\rangle}\left[A_{u},\langle e \mid x\rangle\right] \frac{1}{\langle e \mid x\rangle}\left(\frac{i}{2} \tilde{X}_{e}-1\right)+\frac{1}{\langle e \mid x\rangle}\left[A_{u}, \frac{i}{2} \tilde{X}_{e}\right] \\
= & -\frac{1}{\langle e \mid x\rangle}\left[A_{u},\langle e \mid x\rangle\right] \hat{h}-\frac{1}{\langle e \mid x\rangle}\left[\frac{\langle u \mid x\rangle}{\langle e \mid x\rangle}, \frac{i}{2} \tilde{X}_{e}\right]\langle e \mid x\rangle \hat{h} \\
= & -\frac{1}{\langle e \mid x\rangle}\left[A_{u},\langle e \mid x\rangle\right] \hat{h} \\
& -\frac{1}{\langle e \mid x\rangle}\left(\left[\langle u \mid x\rangle, \frac{i}{2} \tilde{X}_{e}\right]-\frac{\langle u \mid x\rangle}{\langle e \mid x\rangle}\left[\langle e \mid x\rangle, \frac{i}{2} \tilde{X}_{e}\right]\right) \hat{h} \\
= & -\frac{1}{\langle e \mid x\rangle}\left[A_{u},\langle e \mid x\rangle\right] \hat{h}+\frac{1}{\langle e \mid x\rangle}\left(\tilde{L}_{u}-\frac{\langle u \mid x\rangle}{\langle e \mid x\rangle} \tilde{L}_{e}\right) \hat{h} \\
= & 0 .
\end{aligned}
$$

Since $A_{u}=\frac{i}{2} \tilde{X}_{u}-\langle u \mid x\rangle \hat{h}$, we have

$$
\begin{aligned}
& {\left[A_{u}, A_{v}\right]=\left[\frac{i}{2} \tilde{X}_{u},-\langle v \mid x\rangle \hat{h}\right]-\left[\frac{i}{2} \tilde{X}_{v},-\langle u \mid x\rangle \hat{h}\right]+[\langle u \mid x\rangle \hat{h},\langle v \mid x\rangle \hat{h}]} \\
& =\left[\frac{i}{2} \tilde{X}_{u},-\langle v \mid x\rangle \hat{h}\right]-\langle v \mid x\rangle[\hat{h},\langle u \mid x\rangle] \hat{h}-\langle u \leftrightarrow v\rangle \\
& =\left[\frac{i}{2} \tilde{X}_{u},-\langle v \mid x\rangle\right] \hat{h}-\langle v \mid x\rangle\left[\frac{i}{2} \tilde{X}_{u}, \hat{h}\right]-\langle v \mid x\rangle[\hat{h},\langle u \mid x\rangle] \hat{h} \\
& =-\tilde{S}_{u v} \hat{h}+\frac{\langle v \mid x\rangle}{\langle e \mid x\rangle}\left[\frac{i}{2} \tilde{X}_{u},\langle e \mid x\rangle\right] H-\frac{\langle v \mid x\rangle}{\langle e \mid x\rangle}\left[\frac{i}{2} \tilde{X}_{e},\langle u \mid x\rangle\right] \hat{h} \\
& -\langle u \leftrightarrow v> \\
& =-\tilde{S}_{u v} \hat{h}+\frac{\langle v \mid x\rangle}{\langle e \mid x\rangle} \tilde{L}_{u} \hat{h}-\frac{\langle v \mid x\rangle}{\langle e \mid x\rangle} \tilde{L}_{u} \hat{h}-\langle u \leftrightarrow v> \\
& =-2 L_{u, v} \hat{h}=-2 \hat{h} L_{u, v} \text {. }
\end{aligned}
$$




\section{Symmetry ANAlysis of the J-KePler Problems}

The goal of this section is to give a detailed dynamical symmetry analysis for the JKepler problem, as a byproduct, we solve the bound state problem for the J-Kepler problem algebraically.

Unless said otherwise, throughout this section we assume that $V$ is a simple euclidean Jordan algebra with rank $\rho \geq 2$ and degree $\delta$. For simplicity, for each $x \in \mathscr{P}$, we shall rewrite $\langle e \mid x\rangle$ as $r$.

8.1. Harmonic Analysis on Projective Spaces. Let us begin with the harmonic analysis on projective space $\mathbb{P}$. Since $\mathbb{P}$ is a real affine variety inside $V$, its coordinate ring $\mathbb{R}[\mathbb{P}]$ is a quotient of the ring of real polynomial functions on $V$. Recall that we use $\Delta_{\mathbb{P}}$ to denote the Laplace operator on $\mathbb{P}$.

Lemma 8.1. Let $V_{m}$ be the set of regular (i.e., polynomial) functions (on $\mathbb{P}$ ) of degree at most $m, \mathcal{V}_{m}$ be the orthogonal complement of $V_{m-1}$ in $V_{m}$. For each $u \in V$, we let $m_{u}$ : $\mathbb{R}[\mathbb{P}] \rightarrow \mathbb{R}[\mathbb{P}]$ denote the multiplication by $\langle u \mid x\rangle$.

i) For any integer $k \geq 0$, there is a $u \in V$ perpendicular to e such that

$$
\tilde{m}_{u}: \mathcal{V}_{k} \stackrel{m_{u}}{\rightarrow} V_{k+1} \stackrel{\pi}{\rightarrow} \mathcal{V}_{k+1}
$$

is nonzero. Here, $\pi$ is the orthogonal projection.

ii) $\mathcal{V}_{m}^{\mathbb{C}}:=\mathcal{V}_{m} \otimes_{\mathbb{R}} \mathbb{C}$ is the $m$-th eigenspace of $\Delta_{\mathbb{P}}$ with eigenvalue $-m\left(m+\frac{\rho \delta}{2}-1\right)$, and the Hilbert space of square integrable complex-valued functions on $\mathbb{P}$ admits an orthogonal decomposition into the eigenspaces of $\Delta_{\mathbb{P}}$ :

$$
L^{2}(\mathbb{P})=\hat{\bigoplus}_{m \geq 0} \mathcal{V}_{m}^{\mathbb{C}}
$$

Proof. It is clear that

$$
V_{m}=\bigoplus_{k=0}^{m} \mathcal{V}_{k}, \quad \mathbb{R}[\mathbb{P}]=\bigoplus_{k=0}^{\infty} \mathcal{V}_{k}
$$

i) Let $\{e\} \cup\left\{e_{i} \mid 1 \leq i \leq \operatorname{dim} V-1\right\}$ be an orthonomal basis for $V$ and $x_{i}=\left\langle e_{i} \mid x\right\rangle$. Since $m_{u}$ maps $V_{k}$ to $V_{k+1}$ for each integer $k \geq 0$, we have the resulting map

$$
\overline{m_{u}}: V_{k} / V_{k-1} \rightarrow V_{k+1} / V_{k} .
$$

For any $\bar{f} \in V_{k+1} / V_{k}$, if we write $\bar{f}=\sum_{i>0} \overline{x_{i} g_{i}}$, we have $\bar{f}=\sum_{i>0} \overline{m_{e_{i}}}\left(\overline{g_{i}}\right)$. In view of the commutative diagram

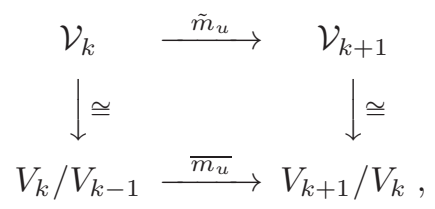

we have

$$
\mathcal{V}_{k+1}=\sum_{i>0} \tilde{m}_{e_{i}}\left(\mathcal{V}_{k}\right)
$$

for any $k \geq 0$. Suppose that $\tilde{m}_{e_{i}}: \mathcal{V}_{m} \rightarrow \mathcal{V}_{m+1}$ is zero for any $i>0$, then $\mathcal{V}_{m+1}=0$ per Eq. 8.2, so $\mathcal{V}_{n}=0$ for any $n \geq m+1$ per Eq. 8.2), then

$$
\mathbb{R}[\mathbb{P}]=\lim _{k \rightarrow \infty} V_{k}=V_{m}
$$

a contradiction. 
ii) Let $u_{1}, \ldots, u_{m}$ be in $V$, and write $x_{u_{i}}$ for $\left\langle u_{i} \mid x\right\rangle, u_{i}^{0}$ for $\left\langle e \mid u_{i}\right\rangle$. Since

$$
\left[\Delta_{\mathbb{P}}, x_{u_{1}} \cdots x_{u_{m}}\right]=\sum_{i=1}^{m} x_{u_{1}} \cdots x_{u_{i-1}}\left[\Delta_{\mathbb{P}}, x_{u_{i}}\right] x_{u_{i+1}} \cdots x_{u_{m}}
$$

in view of Eq. 6.7] and part ii) of Lemma6.1, we have

$$
\begin{aligned}
\Delta_{\mathbb{P}}\left(x_{u_{1}} \cdots x_{u_{m}}\right)= & \sum_{i=1}^{m} x_{u_{1}} \cdots x_{u_{i-1}}\left[\Delta_{\mathbb{P}}, x_{u_{i}}\right]\left(x_{u_{i+1}} \cdots x_{u_{m}}\right) \\
= & \sum_{i=1}^{m} x_{u_{1}} \cdots x_{u_{i-1}}\left(-2 r \tilde{L}_{u_{i}}+2 x_{u_{i}} \tilde{L}_{e}\right)\left(x_{u_{i+1}} \cdots x_{u_{m}}\right) \\
= & -m \frac{\rho \delta}{2} x_{u_{1}} \cdots x_{u_{m}}+\frac{\rho \delta}{2} r u_{i}^{0} \sum_{i=1}^{m} x_{u_{1}} \cdots \hat{x}_{u_{i}} \cdots x_{u_{m}} \\
& +\sum_{i=1}^{m} x_{u_{1}} \cdots x_{u_{i-1}}\left(-2 r \hat{L}_{u_{i}}+2 x_{u_{i}} \hat{L}_{e}\right)\left(x_{u_{i+1}} \cdots x_{u_{m}}\right) \\
= & -\left(m \frac{\rho \delta}{2}+m(m-1)\right) x_{u_{1}} \cdots x_{u_{m}}+\frac{\rho \delta}{2} r \sum_{i=1}^{m} u_{i}^{0} x_{u_{1}} \cdots \hat{x}_{u_{i}} \cdots x_{u_{m}} \\
& -2 r \sum_{i=1}^{m} x_{u_{1}} \cdots x_{u_{i-1}} \hat{L}_{u_{i}}\left(x_{u_{i+1}} \cdots x_{u_{m}}\right) \\
\equiv & -m\left(m+\frac{\rho \delta}{2}-1\right) x_{u_{1}} \cdots x_{u_{m}} \quad\left(\bmod V_{m-1}\right)
\end{aligned}
$$

because $r=\sqrt{2 / \rho}$ on $\mathbb{P}$.

It is then clear that $\Delta_{\mathbb{P}}$ maps $V_{m}$ into $V_{m}$ for each $m \geq 0$ and resulting map

$$
\overline{\Delta_{\mathbb{P}}}: V_{m} / V_{m-1} \rightarrow V_{m} / V_{m-1}
$$

is the scalar multiplication by $-m\left(m+\frac{\rho \delta}{2}-1\right)$. Since $\Delta_{\mathbb{P}}$ is a hermitian operator and maps $V_{m-1}$ into $V_{m-1}, \Delta_{\mathbb{P}}$ maps $\mathcal{V}_{m}$ into $\mathcal{V}_{m}$, so we have commutative diagram

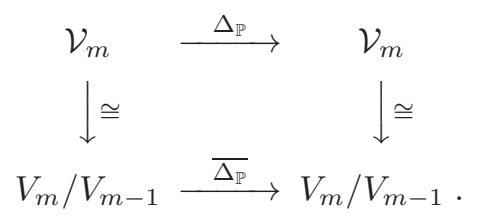

Since $\mathcal{V}_{m} \neq\{0\}$ per part i), we conclude that $\mathcal{V}_{m}$ is an eigenspace of $\Delta_{\mathbb{P}}$ with eigenvalue $-m\left(m+\frac{\rho \delta}{2}-1\right)$.

Since the ring of regular functions is dense in the ring of real continuous functions and

$$
\mathbb{R}[\mathbb{P}]=\bigoplus_{k=0}^{\infty} \mathcal{V}_{k},
$$

we have

$$
L^{2}(\mathbb{P})=\hat{\bigoplus}_{m \geq 0} \mathcal{V}_{m}^{\mathbb{C}}
$$


8.2. Associated Lagueree Polynomials. Here, we give a quick review of the associated Lagueree polynomials. Let $\alpha$ be a real number and $n \geq 0$ be an integer. By definition, the associated Lagueree polynomial $L_{n}^{\alpha}(x)$ is the polynomial solution of equation

$$
x y^{\prime \prime}+(\alpha+1-x) y^{\prime}+n y=0
$$

whose the leading coefficient is $(-1)^{n} \frac{1}{n !}$. We note that $L_{n}^{\alpha}(x)$ has degree $n$, for example,

$$
\begin{aligned}
L_{0}^{\alpha}(x) & =1 \\
L_{1}^{\alpha}(x) & =-x+\alpha+1 \\
L_{2}^{\alpha}(x) & =\frac{1}{2} x^{2}-(\alpha+2) x+\frac{1}{2}(\alpha+1)(\alpha+2) \\
& \vdots
\end{aligned}
$$

In general, we have

$$
L_{n}^{\alpha}(x)=\frac{x^{-\alpha} e^{x}}{n !} \frac{d^{n}}{d x^{n}}\left(e^{-x} x^{n+\alpha}\right) .
$$

It is a fact that $L_{n}^{\alpha}(x)$ 's form an orthogonal basis for $L^{2}\left(\mathbb{R}_{+}, x^{\alpha} e^{-x} d x\right)$ :

$$
\int_{0}^{\infty} x^{\alpha} e^{-x} L_{n}^{\alpha}(x) L_{m}^{\alpha}(x) d x=\frac{\Gamma(n+\alpha+1)}{n !} \delta_{m n} ;
$$

moreover, a degree $n$ polynomial in $x$ can be uniquely written as a linear combination of $L_{k}^{\alpha}(x)$ with $0 \leq k \leq n$.

It is then clear that,

$$
\begin{gathered}
\text { for any integer } l \geq 0, x^{l-\frac{(\rho / 2-1) \delta}{2}} e^{-x} L_{n}^{2 l+\frac{\rho \delta}{2}-1}(2 x) \text { 's form } \\
\text { an orthogonal basis for } L^{2}\left(\mathbb{R}_{+}, x^{(\rho-1) \delta-1} d x\right) .
\end{gathered}
$$

It is also a fact that

$$
n L_{n}^{\alpha}(x)=(n+\alpha) L_{n-1}^{\alpha}(x)-x L_{n-1}^{\alpha+1}(x)
$$

and

$$
L_{n+1}^{\alpha}(x)=\frac{1}{n+1}\left((2 n+1+\alpha-x) L_{n}^{\alpha}(x)-(n+\alpha) L_{n-1}^{\alpha}(x)\right) .
$$

8.3. Hidden Harmonic Analysis on Kepler Cones. For each integer $l \geq 0$, we fix an orthonormal spanning set $\left\{Y_{l m} \mid m \in \mathcal{I}(l)\right\}$ for $\mathcal{V}_{l}$. Note that each $Y_{l m}$ can be represented by a homogeneous degree $l$-polynomial in $x$, which will be denoted by $Y_{l m}(x)$. For integer $k \geq 1$, we introduce

$$
\varphi_{k l m}(x):=r^{-\frac{(\rho / 2-1) \delta}{2}} L_{k-1}^{2 l+\frac{\rho \delta}{2}-1}(2 r) e^{-r} Y_{l m}(x)
$$

where $r=\langle e \mid x\rangle$. One can verify that $\varphi_{k l m}$ is square integrable with respect to $\frac{1}{r} \operatorname{vol} \mathscr{P}$ :

$$
\begin{aligned}
\left(\frac{2}{\rho}\right)^{l} \int_{\mathscr{P}}\left|\varphi_{k l m}\right|^{2} \frac{1}{r} \operatorname{vol} \mathscr{P} & =\int_{\mathbb{P}}\left|Y_{l m}\right|^{2} \operatorname{vol}_{\mathbb{P}} \cdot \\
& \cdot \int_{0}^{\infty} r^{2 l-(\rho / 2-1) \delta} \cdot\left(L_{k-1}^{2 l+\frac{\rho \delta}{2}-1}(2 r)\right)^{2} \cdot r^{(\rho-1) \delta-1} e^{-2 r} d r \\
= & \int_{0}^{\infty} r^{2 l+\frac{\rho \delta}{2}-1}\left(L_{k-1}^{2 l+\frac{\rho \delta}{2}-1}(2 r)\right)^{2} e^{-2 r} d r \\
= & \frac{\Gamma\left(2 l+\frac{\rho \delta}{2}-1+k\right)}{2^{2 l+\rho \delta / 2}(k-1) !}<\infty
\end{aligned}
$$

because $\delta \geq 1$ and $\rho \geq 1$. 
Let $H_{0}:=-\frac{i}{2}\left(X_{e}+Y_{e}\right)$, then

$$
\tilde{H}_{0}=\frac{1}{2}\left(\frac{\hat{L}_{e}^{2}-\left(2 \lambda_{e}-1\right) \hat{L}_{e}+\Delta_{\mathbb{P}}+B}{r}-r\right) .
$$

We say that a smooth nonzero function $\varphi$ on the Kepler cone is an eigenfunction of $\tilde{H}_{0}$ if it is square integrable with respect to $\frac{1}{r} \mathrm{vol}_{\mathscr{P}}$ and satisfies equation

$$
\tilde{H}_{0} \varphi=\lambda \varphi
$$

for some real number $\lambda$. With the help of part ii) of Lemma 8.1 and Eq. 8.3), one can check that

$$
\tilde{H}_{0} \varphi_{k l m}=-\left(l+k-1+\frac{\rho \delta}{4}\right) \varphi_{k l m},
$$

so $\varphi_{k l m}$ is an eigenfunction of $\tilde{H}_{0}$ with eigenvalue $-\left(l+k-1+\frac{\rho \delta}{4}\right)$.

Let $\mathscr{V}_{l}(k):=\operatorname{span}_{\mathbb{C}}\left\{\varphi_{k l m} \mid m \in \mathcal{I}(l)\right\}$ for each integer $l \geq 0$ and $k \geq 1$, and

$$
\tilde{\mathscr{H}}_{I}:=\bigoplus_{l=0}^{I} \mathscr{V}_{l}(I+1-l)
$$

for each integer $I \geq 0$. Finally, we let $\tilde{\mathcal{H}}:=\bigoplus_{I=0}^{\infty} \tilde{\mathscr{H}}_{I}$ and $\pi(\mathcal{O}):=\tilde{\mathcal{O}}$ for any $\mathcal{O}$ in the conformal algebra. In view of statement (8.6), it is clear that $\varphi_{\mathrm{klm}}$ 's form an orthogonal basis for $\tilde{\mathcal{H}}$.

Proposition 8.1. $i) \tilde{\mathscr{H}}_{I}$ is the eigenspace of $\tilde{H}_{0}$ with eigenvalue $-(I+\rho \delta / 4)$ and

$$
L^{2}\left(\mathscr{P}, \frac{1}{r} \operatorname{vol} \mathscr{P}\right)=\hat{\bigoplus}_{I=0}^{\infty} \tilde{\mathscr{H}}_{I} .
$$

Moreover, $\varphi_{k l m}$ 's form an orthogonal basis for $L^{2}\left(\mathscr{P}, \frac{1}{r} \operatorname{vol} \mathscr{P}\right)$.

ii) $(\pi, \tilde{\mathcal{H}})$ is a unitary representation of the conformal algebra.

iii) $\left(\left.\pi\right|_{\overline{\mathfrak{u}}}, \tilde{\mathscr{H}}_{I}\right)$ is an irreducible representation of $\overline{\mathfrak{u}}$. Consequently $\left(\left.\pi\right|_{\mathfrak{u}}, \tilde{\mathscr{H}}_{I}\right)$ is an irreducible representation of $\mathfrak{u}$.

iv) $(\pi, \tilde{\mathcal{H}})$ is a unitary lowest weight representation of the conformal algebra with lowest weight equal to $\frac{\delta}{2} \lambda_{0}$. Here $\lambda_{0}$ is the fundamental weight conjugate to the unique noncompact simple root $\alpha_{0}$ in Lemma 4.1 i.e., $\lambda_{0}\left(H_{\alpha_{i}}\right)=0$ for $i>0$ and

$$
2 \frac{\lambda_{0}\left(H_{\alpha_{0}}\right)}{\alpha_{0}\left(H_{\alpha_{0}}\right)}=1
$$

Proof. i) By virtue of Theorem II.10 of Ref. [11], we have

$$
L^{2}\left(\mathscr{P}, \frac{1}{r} \operatorname{vol}_{\mathscr{P}}\right)=L^{2}\left(\mathbb{R}_{+}, r^{(\rho-1) \delta-1} d r\right) \otimes L^{2}(\mathbb{P}) .
$$

Then

$$
\begin{aligned}
L^{2}\left(\mathscr{P}, \frac{1}{r} \operatorname{vol} \mathscr{P}\right) & =\hat{\bigoplus}_{l=0}^{\infty}\left(L^{2}\left(\mathbb{R}_{+}, r^{(\rho-1) \delta-1} d r\right) \otimes \mathcal{V}_{l}^{\mathbb{C}}\right) \quad \text { Eq. [8.1) } \\
& =\hat{\bigoplus}_{l=0}^{\infty} \hat{\bigoplus}_{k=1}^{\infty} \bigoplus_{m \in \mathcal{I}(l)} \operatorname{span}_{\mathbb{C}}\left\{\varphi_{k l m}\right\} \quad \text { statement [8.6) } \\
& =\hat{\bigoplus}_{I=0}^{\infty} \tilde{\mathscr{H}}_{I} .
\end{aligned}
$$

Therefore, we conclude that $\left\{\varphi_{k l m}\right\}$ is an orthogonal basis for $L^{2}\left(\mathscr{P}, \frac{1}{r} \operatorname{vol} \mathscr{P}\right)$ and $\tilde{\mathscr{H}}_{I}$ is the $I$-th eigenspace of $\tilde{H}_{0}$ with eigenvalue $-(I+\rho \delta / 4)$. 
ii) First, we need to show that $\tilde{\mathcal{O}}(\psi) \in \tilde{\mathcal{H}}$ for any $\mathcal{O} \in \mathfrak{c o}$ and any $\psi \in \tilde{\mathcal{H}}$. Without loss of generality we may assume that $\mathcal{O}$ is $L_{u}, X_{e}$ or $Y_{e}$ and

$$
\psi=\varphi_{k l m}=r^{-\frac{(\rho / 2-1) \delta}{2}} L_{k}^{2 l+\frac{\rho \delta}{2}-1}(2 r) e^{-r} Y_{l m}(x) .
$$

Using Eq. 8.8) one can see that $\tilde{Y}_{e}\left(\varphi_{k l m}\right) \in \tilde{\mathcal{H}}$. Then

$$
\begin{aligned}
\tilde{X}_{e}\left(\varphi_{k l m}\right) & =\left(2 \sqrt{-1} \tilde{H}_{0}-\tilde{Y}_{e}\right)\left(\varphi_{k l m}\right) \\
& =2 \sqrt{-1}\left(k+l-1+\frac{\rho \delta}{2}\right) \varphi_{k l m}-\tilde{Y}_{e}\left(\varphi_{k l m}\right) \\
& \in \tilde{\mathcal{H}} .
\end{aligned}
$$

Since

$$
\begin{aligned}
\hat{L}_{u}\left(\varphi_{k l m}\right)= & \hat{L}_{u}\left(r^{-\frac{(\rho / 2-1) \delta}{2}} L_{k}^{2 l+\frac{\rho \delta}{2}-1}(2 r) e^{-r}\right) Y_{l m}(x) \\
& +r^{-\frac{(\rho / 2-1) \delta}{2}} L_{k}^{2 l+\frac{\rho \delta}{2}-1}(2 r) e^{-r} \hat{L}_{u}\left(Y_{l m}(x)\right),
\end{aligned}
$$

one can see that $\hat{L}_{u}\left(\varphi_{k l m}\right) \in \bigoplus_{k^{\prime} \leq k+l+1, l^{\prime} \leq l+1} \mathscr{V}_{l^{\prime}}\left(k^{\prime}\right)$. It is also clear that

$$
\lambda_{u} \cdot \varphi_{k l m} \in \bigoplus_{k^{\prime} \leq k+l+1, l^{\prime} \leq l+1} \mathscr{V}_{l^{\prime}}\left(k^{\prime}\right),
$$

so $\tilde{L}_{u}\left(\varphi_{k l m}\right) \in \tilde{\mathcal{H}}$.

Next, we verify that

$$
\left(\varphi_{k l m}, \tilde{\mathcal{O}}\left(\varphi_{k^{\prime} l^{\prime} m^{\prime}}\right)\right)+\left(\tilde{\mathcal{O}}\left(\varphi_{k l m}\right), \varphi_{k^{\prime} l^{\prime} m^{\prime}}\right)=0
$$

for $\mathcal{O} \in \mathfrak{c o}$. We may assume that $\mathcal{O}$ is $L_{u}, X_{e}$ or $Y_{e}$. It is clearly $\mathrm{OK}$ when $\mathcal{O}=Y_{e}$ because $\tilde{Y}_{e}=-i r$. Since $\tilde{X}_{e}=2 i \tilde{H}_{0}-\tilde{Y}_{e}$, to show that $\tilde{X}_{e}$ is anti-hermitian, it suffices to verify that $\left(\varphi_{k l m}, \tilde{H}_{0}\left(\varphi_{k^{\prime} l^{\prime} m^{\prime}}\right)\right)-\left(\tilde{H}_{0}\left(\varphi_{k l m}\right), \varphi_{k^{\prime} l^{\prime} m^{\prime}}\right)=0$ or $\left(k^{\prime}+l^{\prime}-k-l\right)\left(\varphi_{k l m}, \varphi_{k^{\prime} l^{\prime} m^{\prime}}\right)=$ 0 , which is obviously true.

To verify that $\left(\varphi_{k l m}, \dot{\tilde{L}}_{u}\left(\varphi_{k^{\prime} l^{\prime} m^{\prime}}\right)\right)+\left(\tilde{L}_{u}\left(\varphi_{k l m}\right), \varphi_{k^{\prime} l^{\prime} m^{\prime}}\right)=0$, in view of part iii) of Lemma5.1 we know that $\left(\varphi_{k l m}, \tilde{L}_{u}\left(\varphi_{k^{\prime} l^{\prime} m^{\prime}}\right)\right)+\left(\tilde{L}_{u}\left(\varphi_{k l m}\right), \varphi_{k^{\prime} l^{\prime} m^{\prime}}\right)$ is equal to

$$
\int_{\mathscr{P}} \mathscr{L}_{u}\left(\overline{\varphi_{k l m}} \varphi_{k^{\prime} l^{\prime} m^{\prime}} \frac{1}{r} \operatorname{vol} \mathscr{P}\right)=\int_{\mathscr{P}} d \iota_{\hat{L}_{u}}\left(\overline{\varphi_{k l m}} \varphi_{k^{\prime} l^{\prime} m^{\prime}} \frac{1}{r} \operatorname{vol} \mathscr{P}\right)=0 .
$$

That is because $\iota_{\hat{L}_{u}}\left(\overline{\varphi_{k l m}} \varphi_{k^{\prime} l^{\prime} m^{\prime}} \frac{1}{r} \operatorname{vol} \mathscr{P}\right)$ approaches to zero exponentially fast as $r \rightarrow \infty$ and approaches to zero as $r \rightarrow 0$, uniformly with respect to the angle directions.

iii) First, we verify that $\tilde{\mathscr{H}}_{I}$ is invariant under the action of $\overline{\mathfrak{u}}$. To see this, we note that $\tilde{\mathscr{H}}_{I}$ is an eigenspace of $\tilde{H}_{0}$, moreover, as operators on Hilbert space $L^{2}\left(\mathscr{P}, \frac{1}{r}\right.$ vol $\left.\mathscr{P}\right), \tilde{H}_{0}$ commutes with $\tilde{\mathcal{O}}$ for any $\mathcal{O} \in \overline{\mathfrak{u}}$.

Since $\tilde{\mathscr{H}}_{I}=\bigoplus_{l=0}^{I} \mathscr{\mathscr { H }}_{l}(I+1-l)$, if the action of $\overline{\mathfrak{u}}$ on $\tilde{\mathscr{H}}_{I}$ were not irreducible, there would be an integer $l$ with $0 \leq l<I$ such that $\left(\psi_{l}, \tilde{\mathcal{O}}\left(\psi_{l+1}\right)\right)=0$ for any $\psi_{l} \in \mathscr{V}_{l}(I+$ $1-l), \psi_{l+1} \in \mathscr{V} / l+1(I-l)$, and any $\mathcal{O} \in \overline{\mathfrak{k}}$.

In view of part i) of Lemma 8.1, we can choose a $u \in V$ with $u \perp e$ such that $\tilde{m}_{u}$ : $\mathcal{V}_{l} \rightarrow \mathcal{V}_{l+1}$ is nontrivial; so there is a $Y_{l m} \in \mathcal{V}_{l}$ and a $Y_{(l+1) m^{\prime}} \in \mathcal{V}_{l+1}$ such that

$$
\int_{\mathbb{P}} Y_{(l+1) m^{\prime}} \cdot \tilde{m}_{u}\left(Y_{l m}\right) \operatorname{vol}_{\mathbb{P}} \neq 0 .
$$

Let

$$
\begin{aligned}
\psi_{l}(x) & =r^{-\frac{(\rho / 2-1) \delta}{2}} L_{k}^{2 l+\frac{\rho \delta}{2}-1}(2 r) e^{-r} Y_{l m}(x), \\
\psi_{l+1}(x) & =r^{-\frac{(\rho / 2-1) \delta}{2}} L_{k-1}^{2 l+\frac{\rho \delta}{2}+1}(2 r) e^{-r} Y_{(l+1) m^{\prime}}(x), \\
\mathcal{O} & =X_{u}+Y_{u} .
\end{aligned}
$$


Then $\mathcal{O} \in \overline{\mathfrak{u}}$ because $u \perp e$. Since $\tilde{\mathcal{O}}=\left[\tilde{L}_{u}, \tilde{X}_{e}-\tilde{Y}_{e}\right]=\left[2 i \tilde{L}_{u}, \tilde{H}_{0}\right]+2 \tilde{Y}_{u}$, we have $\left(\psi_{l}, \tilde{\mathcal{O}}\left(\psi_{l+1}\right)\right)=\left(\psi_{l}, 2 \tilde{Y}_{u} \cdot \psi_{l+1}\right)$; so, in view of Eq. [8.11), $\left(\psi_{l}, \tilde{\mathcal{O}}\left(\psi_{l+1}\right)\right)=0$ would imply that

$$
\int_{0}^{\infty} x^{\alpha+2} e^{-x} L_{k}^{\alpha}(x) L_{k-1}^{\alpha+2}(x) d x=0 .
$$

where $\alpha=2 l+\rho \delta / 2-1$. But that is a contradiction: using Eq. 8.7), one can show that

$$
\int_{0}^{\infty} x^{\alpha+2} e^{-x} L_{k}^{\alpha}(x) L_{k-1}^{\alpha+2}(x) d x=-2 \frac{\Gamma(k+\alpha+2)}{(k-1) !} \neq 0 .
$$

iv) Let us take the simple root system $\alpha_{0}, \ldots, \alpha_{r}$ specified in Lemma4.1 and

$$
\psi_{0}(x)=r^{-\frac{(\rho / 2-1) \delta}{2}} e^{-r} .
$$

Since $\tilde{\mathscr{H}}_{0}\left(=\operatorname{span}_{\mathbb{C}}\left\{\psi_{0}\right\}\right)$ is one dimensional and $\overline{\mathfrak{u}}$ is semi-simple, the action of $\overline{\mathfrak{u}}^{\mathbb{C}}$ on $\tilde{\mathscr{H}}_{0}$ must be trivial. Therefore, for $i \geq 1$, in view of the fact that $E_{ \pm \alpha_{i}}, H_{\alpha_{i}} \in \overline{\mathfrak{u}}^{\mathbb{C}}$, we have

$$
\tilde{E}_{-\alpha_{i}} \psi_{0}=0, \quad \tilde{H}_{\alpha_{i}} \psi_{0}=0 .
$$

On the other hand, since $E_{-\alpha_{0}}=\frac{i}{2}\left(X_{e_{11}}-Y_{e_{11}}\right)+L_{e_{11}}$ and $H_{\alpha_{0}}=i\left(X_{e_{11}}+Y_{e_{11}}\right) \equiv$ $-\frac{2}{\rho} H_{0}(\bmod \overline{\mathfrak{u}})$, by a computation, we have

$$
\tilde{E}_{-\alpha_{0}} \psi_{0}=0, \quad \tilde{H}_{\alpha_{0}} \psi_{0}=\frac{\delta}{2} \psi_{0} .
$$

Therefore, in view of the fact that $\alpha_{0}\left(H_{\alpha_{0}}\right)=2, \psi_{0}$ is a lowest weight state with weight $\frac{\delta}{2} \lambda_{0}$.

Since operator $\tilde{Y}_{v}$ is the multiplication by $-i\langle v \mid x\rangle$, we have

$$
r^{-\frac{(\rho / 2-1) \delta}{2}} e^{-r} \sum_{i_{1}, \ldots, i_{n}} \alpha_{i_{1} \cdots i_{n}} x_{1}^{i_{1}} \cdots x_{n}^{i_{n}}=\left(\sum_{i_{1}, \ldots, i_{n}} \alpha_{i_{1} \cdots i_{n}}\left(i \tilde{Y}_{e_{1}}\right)^{i_{1}} \cdots\left(i \tilde{Y}_{e_{n}}\right)^{i_{n}}\right) \psi_{0},
$$

so the representation $(\pi, \tilde{\mathscr{H}})$ is generated from $\psi_{0}$. Since this representation is unitary, it must be irreducible.

In summary, $(\pi, \tilde{\mathscr{H}})$ is a unitary lowest weight representation with lowest weight $\frac{\delta}{2} \lambda_{0}$.

Remark 8.1. Let $\mathcal{P}$ be the space of regular functions on $\mathscr{P}$, i.e.,

$$
\mathcal{P}=\{p: \mathscr{P} \rightarrow \mathbb{C} \mid p \text { is a polynomial on } V\} .
$$

In view of Eqn. (8.9),

$$
\tilde{D}:=e^{-r} r^{-\frac{(\rho / 2-1) d}{2}} \mathcal{P}
$$

can be taken as a dense common domain of definition for $\tilde{\mathcal{O}}, \mathcal{O} \in \mathfrak{c o}$.

The following main theorem is an easy corollary of the above proposition.

Theorem 4. Let Co be the conformal group of the Jordan algebra with rank at least two, $\mathrm{K}$ be the closed Lie subgroup of Co whose Lie algebra is $\mathfrak{u}, \lambda_{0}$ be the fundamental weight conjugate to the unique non-compact simple root $\alpha_{0}$ in Lemma 4.1] $\tilde{H}_{0}:=-\frac{i}{2}\left(\tilde{X}_{e}+\tilde{Y}_{e}\right)$, $\tilde{\mathscr{H}}_{I}$ be the $I$-th eigenspace of $\tilde{H}_{0}$, and $\tilde{\mathcal{H}}:=\bigoplus_{I=0}^{\infty} \tilde{\mathscr{H}}_{I}$.

1) The hidden action $\pi$ in Theorem $\square$ turns $\tilde{\mathcal{H}}$ into a unitary lowest weight $(\mathfrak{c o}, \mathrm{K})$ module with lowest weight $\frac{\delta}{2} \lambda_{0}$. Here the action is unitary with respect to inner product

$$
\left(\psi_{1}, \psi_{2}\right)=\int_{\mathscr{P}} \bar{\psi}_{1} \psi_{2} \frac{1}{r} \operatorname{vol} \mathscr{P} .
$$


2) The unitary lowest weight representation of $\mathrm{Co}$, whose underlying $(\mathfrak{c o}, \mathrm{K})$-module is the $(\mathfrak{c o}, \mathrm{K})$-module in part 1), can be realized by $L^{2}\left(\mathscr{P}, \frac{1}{r} \operatorname{vol} \mathscr{P}\right)$.

3) Decomposition $\tilde{\mathcal{H}}=\bigoplus_{I=0}^{\infty} \tilde{\mathscr{H}}_{I}$ is a multiplicity free $K$-type formula.

Note that, the unitary lowest weight representation of Co appeared in this theorem is the minimal representation of Co in the sense of A. Joseph [8], and has the smallest positive Gelfand-Kirillov dimension. This theorem has a more general version which takes care of all unitary lowest weight representations of the smallest positive Gelfand-Kirillov dimension. Since it is a refinement of part (ii) of Theorem XIII.3.4 from Ref. [5] for the case $\nu=\frac{\delta}{2}$ there, this theorem can be conceivably generalized to cover the case for a generic $\nu$ there.

8.4. Solution of the J-Kepler Problems. For a J-Kepler problem, we are primarily interested in solving the bound state problem here, i.e., the following (energy) spectrum problem:

$$
\left\{\begin{aligned}
\hat{h} \psi & =E \psi \\
\int_{\mathscr{P}}|\psi|^{2} \operatorname{vol}_{\mathscr{P}} & <\infty, \quad \psi \not \equiv 0 .
\end{aligned}\right.
$$

It turns out that $E$ has to take ceratin discrete values. For example, for the original Kepler problem, we have

$$
E=-\frac{1}{2 n^{2}}, \quad n=1,2, \ldots
$$

The Hilbert space of bound states, denoted by $\mathscr{H}$, is defined to be the completion of the linear span of all eigenfunctions of $\hat{h}$.

Theorem 5. Let $V$ be a simple euclidean Jordan algebra with rank $\rho \geq 2$ and degree $\delta$ and $\mathrm{Co}$ be the conformal group of $V$. For the J-Kepler problem associated to $V$, the following statements are true:

1) The bound state energy spectrum is

$$
E_{I}=-\frac{1 / 2}{\left(I+\frac{\rho \delta}{4}\right)^{2}}
$$

where $I=0,1,2, \ldots$

2) There is a unitary action of $\mathrm{Co}$ on the Hilbert space of bound states, $\mathscr{H}$. In fact, $\mathscr{H}$ provides a realization for the minimal representation of the conformal group Co.

3) The orthogonal decomposition of $\mathscr{H}$ into the energy eigenspaces is just the multiplicity free $K$-type formula for the minimal representation.

Proof. We start with the eigenvalue problem for $\tilde{H}_{0}$ :

$$
\tilde{H}_{0} \tilde{\psi}=-n_{I} \tilde{\psi}
$$

where $n_{I}=(I+\rho \delta / 4)$ and $\tilde{\psi}$ is square integrable with respect to measure $\frac{1}{r} \operatorname{vol} \mathscr{P}$ and $\tilde{\psi} \not \equiv 0$. The above equation can be recast as

$$
-\frac{1}{2}\left(\Delta+\frac{B}{r^{2}}+\frac{2 n_{I}}{r}\right) \tilde{\psi}(x)=-\frac{1}{2} \tilde{\psi}(x) .
$$

Let $\psi(x):=\tilde{\psi}\left(\frac{x}{n_{I}}\right)$, then the preceding equation becomes

$$
\left(-\frac{1}{2} \Delta-\frac{B}{2 r^{2}}-\frac{1}{r}\right) \psi(x)=-\frac{1 / 2}{n_{I}^{2}} \psi(x),
$$


i.e.,

$$
\hat{h} \psi=-\frac{1 / 2}{n_{I}^{2}} \psi .
$$

One can check that $\psi$ is square integrable with respect to measure $\operatorname{vol} \mathscr{P}$. Therefore, $\tilde{\psi}$ is an eigenfunction of $\tilde{H}_{0} \Rightarrow \psi$ is an eigenfunction of $\hat{h}$. By turning the above arguments backward, one can show that the converse of this statement is also true. Therefore,

$$
\tilde{\psi} \text { is an eigenfunction of } \tilde{H}_{0} \Leftrightarrow \psi \text { is an eigenfunction of } \hat{h} \text {. }
$$

Introduce

$$
\mathscr{H}_{I}:=\left\{\psi \mid \tilde{\psi} \in \tilde{\mathscr{H}}_{I}\right\}, \quad \mathcal{H}:=\bigoplus_{i=0}^{\infty} \mathscr{H}_{I},
$$

and denote by $\tau: \mathcal{H} \rightarrow \tilde{\mathcal{H}}$ the linear map such that

$$
\tau(\psi)(x)=n_{I}^{\frac{(\rho-1) \delta}{2}+1} \psi\left(n_{I} x\right)
$$

for $\psi \in \mathscr{H}_{I}$. By virtue of Theorem 2 in Ref. [12], one can show that $\tau$ is an isometry. Here, the inner product on $\mathcal{H}$ is the usual one: for $\psi, \phi$ in $\mathcal{H}$, we have

$$
\langle\psi, \phi\rangle=\int_{\mathscr{P}} \bar{\psi} \phi \operatorname{vol} \mathscr{P} .
$$

Since $\tilde{\mathcal{H}}$ is a unitary lowest weight Harish-Chandra module, and $\tau$ is an isometry, $\mathcal{H}$ becomes a unitary lowest weight Harish-Chandra module. Since the completion of $\mathcal{H}$ is the Hilbert space of bound states, the rest is clear from Theorem 4

$$
\text { Appendix A. Proof of } O(1)=0 \text { And } \mathscr{O}(1)=0
$$

We shall write

$$
X=-\frac{1}{r}(R+\Delta+B)
$$

where $R=\hat{L}_{e}^{2}-((\rho-1) \delta-1) \hat{L}_{e}$ and $\Delta=A \sum_{\alpha, \beta}\left[\hat{L}_{e_{\alpha}}, \hat{L}_{e_{\beta}}\right]^{2}$. The following facts shall be used often in the computations:

$$
\begin{aligned}
{[\Delta,\langle u \mid x\rangle] } & =-2 r \tilde{L}_{u}+2\langle u \mid x\rangle \tilde{L}_{e}, \\
\Delta(\langle u \mid x\rangle) & =-\frac{\rho \delta}{2}(\langle u \mid x\rangle-\langle u \mid e\rangle r), \\
R(f(x)) & =\left(k^{2}+k-(\rho-1) \delta k\right) f(x) \quad \text { if } f(x) \text { has homogeneous degree }-k .
\end{aligned}
$$

Since

$$
\left[\tilde{L}_{u}, X\right]=-\frac{\langle u \mid x\rangle}{r^{2}}(R+\Delta+B)-\frac{1}{r}\left[\hat{L}_{u}, \Delta\right]-\frac{1}{r}\left[\Delta, \lambda_{u}\right]
$$

we have

$$
\begin{aligned}
{\left[\tilde{L}_{u}, X\right](1) } & =-B\langle u \mid x\rangle / r^{2}-\Delta\left(\lambda_{u}\right) / r \\
& =-B\langle u \mid x\rangle / r^{2}+\frac{(\rho / 2-1) \delta}{2 r^{2}}[-\Delta,\langle u \mid x\rangle] \\
& =\left(-B+\frac{\rho(\rho-2) \delta^{2}}{8}\right) \frac{\langle x \mid u\rangle}{r^{2}}-\frac{\rho(\rho-2) \delta^{2}}{8} \frac{\langle u \mid e\rangle}{r} .
\end{aligned}
$$

We may further assume that $\langle u \mid e\rangle=\langle v \mid e\rangle=0$ in the computations below. 
Proof of $O(1)=0$. Since

$$
\begin{aligned}
& {\left[\left[\tilde{L}_{u}, X\right], \lambda_{v}\right](1)=\left[\left[\tilde{L}_{u}, \lambda_{v}\right], X\right](1)+\left[\left[\lambda_{v}, X\right], \tilde{L}_{u}\right](1)} \\
& =\left[\hat{L}_{u}\left(\lambda_{v}\right), X\right](1)+\left[\frac{1}{r}\left[\Delta, \lambda_{v}\right], \tilde{L}_{u}\right](1) \\
& =\frac{1}{r}\left[\Delta, \hat{L}_{u}\left(\lambda_{v}\right)\right](1)+(\rho / 2-1) \delta\left[-\frac{1}{r} \tilde{L}_{v}+\frac{\langle x \mid v\rangle}{r} \tilde{L}_{e}, \tilde{L}_{u}\right](1) \\
& =\frac{1}{r} \Delta\left(\hat{L}_{u}\left(\lambda_{v}\right)\right) \\
& +(\rho / 2-1) \delta\left(\left[\frac{-1}{r}, \tilde{L}_{u}\right]\left(-\lambda_{v}\right)+\left[\tilde{L}_{u}, \frac{\langle x \mid v\rangle}{r^{2}}\right]\left(\lambda_{e}\right)\right) \\
& =\frac{(\rho-2) \delta}{4 r} \Delta\left(-\frac{\langle x \mid u v\rangle}{(r)}+\frac{\langle x \mid u\rangle\langle x \mid v\rangle}{r^{2}}\right) \\
& -\frac{(\rho-2)(\rho-1) \delta^{2}}{4} \frac{\langle x \mid u v\rangle}{r^{2}} \\
& +(\rho / 2-1) \delta(3 \rho / 4-1 / 2) \delta \frac{\langle x \mid u\rangle\langle x \mid v\rangle}{r^{2}} \\
& =\frac{(\rho-2) \delta}{4 r}\left(\frac{\rho \delta}{2} \frac{\langle x \mid u v\rangle-r\langle u \mid v\rangle}{r}+\frac{1}{r^{2}} \Delta(\langle x \mid u\rangle\langle x \mid v\rangle)\right) \\
& -\frac{(\rho-2)(\rho-1) \delta^{2}}{4} \frac{\langle x \mid u v\rangle}{r^{2}} \\
& +(\rho / 2-1) \delta(3 \rho / 4-1 / 2) \delta \frac{\langle x \mid u\rangle\langle x \mid v\rangle}{r^{2}} \\
& =\frac{(\rho-2) \delta}{4 r^{3}}\left(\left(\frac{\rho \delta}{2}+2\right) r\langle x \mid u v\rangle-(\rho d+2)\langle x \mid u\rangle\langle x \mid v\rangle\right) \\
& -\frac{\rho(\rho-2) \delta^{2}}{8} \frac{\langle u \mid v\rangle}{r}-\frac{(\rho-2)(\rho-1) \delta^{2}}{4} \frac{\langle x \mid u v\rangle}{r^{2}} \\
& +(\rho / 2-1) \delta(3 \rho / 4-1 / 2) d \frac{\langle x \mid u\rangle\langle x \mid v\rangle}{r^{2}} \\
& =\frac{(\rho-2) \delta}{4}\left(\left(\frac{\rho}{2}-1\right) \delta-2\right)\left(-\frac{\langle x \mid u v\rangle}{r^{2}}+\frac{\langle x \mid u\rangle\langle x \mid v\rangle}{r^{2}}\right) \\
& -\frac{\rho(\rho-2) \delta^{2}}{8} \frac{\langle u \mid v\rangle}{r},
\end{aligned}
$$

and

$$
\hat{L}_{v}\left(\left[\tilde{L}_{u}, X\right](1)\right)=\left(-B+\frac{\rho(\rho-2) \delta^{2}}{8}\right) \cdot\left[-\frac{\langle x \mid u v\rangle}{r^{2}}+2 \frac{\langle x \mid u\rangle\langle x \mid v\rangle}{r^{3}}\right],
$$

we have

$$
\begin{aligned}
{\left[\tilde{L}_{v},\left[\tilde{L}_{u}, X\right]\right](1)=} & \tilde{L}_{v}\left(\left[\tilde{L}_{u}, X\right](1)\right)+\left[\tilde{L}_{u}, X\right]\left(\lambda_{v}\right) \\
= & \hat{L}_{v}\left(\left[\tilde{L}_{u}, X\right](1)\right)+\left[\left[\tilde{L}_{u}, X\right], \lambda_{v}\right](1) \\
= & \left(\left(B-\frac{\rho(\rho-2) \delta^{2}}{8}\right)-\frac{(\rho-2) \delta}{4}\left(\left(\frac{\rho}{2}-1\right) \delta-2\right)\right) \frac{\langle x \mid u v\rangle}{r^{2}} \\
& -\frac{\rho(\rho-2) \delta^{2}}{8} \frac{\langle u \mid v\rangle}{r} \\
& +\left(-2 B+\frac{(\rho-2) \delta}{4}\left(\left(\frac{3 \rho}{2}-1\right) \delta-2\right)\right) \frac{\langle x \mid u\rangle\langle x \mid v\rangle}{r^{3}} .
\end{aligned}
$$

On the other hand,

$$
\left[\tilde{L}_{u v}, X\right](1)=\left(-B+\frac{\rho(\rho-2) \delta^{2}}{8}\right) \frac{\langle x \mid u v\rangle}{r^{2}}-\frac{\rho(\rho-2) \delta^{2}}{8} \frac{\langle u \mid v\rangle}{r},
$$


so $\left[\tilde{L}_{v},\left[\tilde{L}_{u}, X\right]\right](1)-\left[\tilde{L}_{u v}, X\right](1)$ is equal to

$$
\begin{aligned}
& \left(2\left(B-\frac{\rho(\rho-2) \delta^{2}}{8}\right)-\frac{(\rho-2) \delta}{4}\left(\left(\frac{\rho}{2}-1\right) \delta-2\right)\right) \frac{\langle x \mid u v\rangle}{r^{2}} \\
+\left(-2 B+\frac{(\rho-2) \delta}{4}\left(\left(\frac{3 \rho}{2}-1\right) \delta-2\right)\right) \frac{\langle x \mid u\rangle\langle x \mid v\rangle}{r^{3}} & \left(-2 B+\frac{(\rho-2) \delta}{4}\left(\left(\frac{3 \rho}{2}-1\right) \delta-2\right)\right)\left(-\frac{\langle x \mid u v\rangle}{r^{2}}+\frac{\langle x \mid u\rangle\langle x \mid v\rangle}{r^{3}}\right) .
\end{aligned}
$$

Then $O(1)=0$ if and only if

$$
B=\frac{\delta}{8}(\rho-2)\left(\left(\frac{3 \rho}{2}-1\right) \delta-2\right) .
$$

Proof of $\mathscr{O}(1)=0$. Since

$$
X\left(\left[\tilde{L}_{v}, X\right](1)\right)=(2-(3 \rho / 2-1) \delta+B)\left(B-\frac{\rho(\rho-2) \delta^{2}}{8}\right) \frac{\langle x \mid v\rangle}{r^{3}},
$$

and

$$
\left[\tilde{L}_{u}, X\right](X(1))=\frac{\langle u \mid x\rangle}{r^{3}}\left[B(2-(\rho-1) \delta+B)+\frac{B \rho \delta}{2}\left(1-\frac{(\rho-2) \delta}{4}\right)\right],
$$

we have

$$
\left[\left[\tilde{L}_{u}, X\right], X\right](1)=\rho \delta\left(B-\frac{d}{8}(\rho-2)\left(\left(\frac{3 \rho}{2}-1\right) d-2\right)\right) \frac{\langle u \mid x\rangle}{r^{3}},
$$

so $\mathscr{O}(1)=0$ if and only if

$$
B=\frac{\delta}{8}(\rho-2)\left(\left(\frac{3 \rho}{2}-1\right) \delta-2\right) .
$$

\section{REFERENCES}

[1] P. Jordan, Z. Phys. 80 (1933), 285.

[2] G. W. Meng, J. Math. Phys. 48 (2007), 032105; G. W. Meng, J. Math. Phys. 49 (2008), 102111; G. W. Meng, J. Math. Phys. 50 (2009), 072107; G. W. Meng, J. Math. Phys. 51 (2010), 122105; J. S. Li and G. W. Meng, to appear.

[3] P. Jordan, J. von Neumann and E. P. Wigner, Ann. Math. 35 (1934), 29.

[4] M. Koecher, S. Walcher and A. Krieg, The Minnesota Notes on Jordan Algebras and Their Applications, Lecture Notes in Mathematics, Vol. $\mathbf{1 7 1 0 .}$

[5] J.Faraut and A.Koranyi, Analysis on Symmetric Cones, Oxford Mathematical Monographs, 1994.

[6] A.O. Barut and H. Kleinert, Phys. Rev. 156 (1967), 1541-1545.

[7] Harish-Chandra, Representations of a semisimple Lie group on a Banach space I, Trans. Amer. Math. Soc. 75 (1953), 185-243.

[8] A. Joseph, Minimal Realizations and Spectrum Generating Algebras, Comm. Math. Phys. 36 (1974), $325-$ 338.

[9] J. Tits, Nederl. Akad. van Wetens. 65 (1962), 530; M. Koecher, Amer. J. Math. 89 (1967) 787; I.L. Kantor, Sov. Math. Dok. 5 (1964), 1404.

[10] G. W. Meng and R. B. Zhang, J. Math. Phys. 52 (2011), 042106; G. W. Meng, J. London Math. Soc. 81 (2010), 663-678.

[11] M. Reed and B. Simon, Methods of Modern Mathematical Physics, Vol. 1, Academic Press Inc., London (1980).

[12] C. Dunkl, A Laguerre Polynomial Orthogonality and the Hydrogen Atom, Analysis and Applications, Vol. 1, No. 2 (2003) 177-188. 
Department of Mathematics, Hong Kong Univ. of Sci. and Tech., Clear Water Bay, KOWLOON, HONG KONG

Current address: Institute for Advanced Study, Einstein Drive, Princeton, New Jersey 08540 USA

E-mail address: mameng@ust.hk 